\title{
PSPACE Bounds for Rank-1 Modal Logics
}

\author{
LUTZ SCHRÖDER \\ DFKI-Lab Bremen and Dept. of Comput. Sci., Universität Bremen \\ and \\ DIRK PATTINSON \\ Department of Computing, Imperial College London
}

For lack of general algorithmic methods that apply to wide classes of logics, establishing a complexity bound for a given modal logic is often a laborious task. The present work is a step towards a general theory of the complexity of modal logics. Our main result is that all rank-1 logics enjoy a shallow model property and thus are, under mild assumptions on the format of their axiomatisation, in PSPACE. This leads to a unified derivation of tight PSPACE-bounds for a number of logics including $K, K D$, coalition logic, graded modal logic, majority logic, and probabilistic modal logic. Our generic algorithm moreover finds tableau proofs that witness pleasant prooftheoretic properties including a weak subformula property. This generality is made possible by a coalgebraic semantics, which conveniently abstracts from the details of a given model class and thus allows covering a broad range of logics in a uniform way.

Categories and Subject Descriptors: F.4.1 [Mathematical Logic and Formal Languages]: Mathematical Logic-Modal Logic; Computational Logic; F.2.2. [Analysis of Algorithms and Problem Complexity]: Nonnumerical Algorithms and Problems-Complexity of Proof Procedures

General Terms: Algorithms, Languages, Theory

Additional Key Words and Phrases: Shallow models, resolution, coalgebra

\section{INTRODUCTION}

Modal logics are attractive from a computational point of view, as they often combine expressiveness with decidability. For many modal logics not involving dynamic features, satisfiability is known to be in PSPACE. This is typically proved for one logic at a time, e.g. by modifications of the witness algorithm for the modal logic $K$ [Ladner 1977; Blackburn et al. 2001], but also using markedly different methods such as the constraint-based PSPACE-algorithm for graded modal logic [Tobies 2001]. Vardi [1989] gives a first glimpse of a generalisable method, equipping various epistemic logics with a neighbourhood frame semantics and showing them to be in $N P$ and $P S P A C E$, respectively (with the $K$ axiom being responsible for PSPACE-hardness; recent work by Halpern and Rêgo [2007] shows that negative introspection brings the complexity back down to $N P$ ). Nevertheless, there is to date no generally applicable theorem that allows establishing PSPACE-bounds for large classes of modal logics in a uniform way.

Here, we generalise the methods of [Vardi 1989] to obtain PSPACE bounds for rank-1 modal logics, i.e. logics axiomatisable by formulas whose modal depth uniformly equals one, in a systematic way. Although presently limited to rank 1, our approach covers numerous relevant and non-trivial examples. We recover known $P S P A C E$ bounds not only for normal modal logics such as $K$ and $K D$, but most notably also for a range of non-normal modal logics such as graded modal logic [Fine 
1972], coalition logic [Pauly 2002], and probabilistic modal logic [Larsen and Skou 1991; Heifetz and Mongin 2001]. Moreover, our methods lead to a previously unknown PSPACE upper bound for majority logic [Pacuit and Salame 2004] that was independently discovered by Demri and Lugiez [2006] at the same time. These logics are far from exotic: graded modal logic plays a role e.g. in decision support and knowledge representation [van der Hoek and Meyer 1992; Ohlbach and Koehler 1999], and probabilistic modal logic has appeared in connection with model checking [Larsen and Skou 1991] and in modelling economic behaviour [Heifetz and Mongin 2001].

The key to such a degree of generality is to parametrise the theory over the type of systems defining the semantics, using coalgebraic methods. Coalgebra conveniently abstracts from the details of a concrete class of models as it encapsulates the precise nature of models in an endofunctor on the category of sets. As specific instances, one obtains e.g. (serial) Kripke frames, (monotone) neighbourhood frames [Hansen and Kupke 2004], game frames [Pauly 2002], probabilistic transition systems and automata [Rabin 1963; Bartels et al. 2004], weighted automata, linear automata [Carlyle and Paz 1971], and multigraphs [D'Agostino and Visser 2002]. Despite the broad range of systems covered by the coalgebraic approach, a substantial body of concepts and non-trivial results has emerged, encompassing e.g. generic notions of bisimilarity and coinduction [Bartels 2003], corecursion [Turi and Plotkin 1997], duality, and ultrafilter extensions [Kupke et al. 2005]. On the applications side, coalgebraic modal logic features in actual specification languages such as the object oriented specification language CCSL [Rothe et al. 2001] and CoCAsL [Mossakowski et al. 2006].

The coalgebraic study of computational aspects of modal logic was initiated in [Schröder 2007], where the finite model property and associated NEXPTIMEbounds were proved. Here, we push these results further and present a shallow model property based on coalgebraic semantics. This leads to a generic PSPACEalgorithm for deciding satisfiability that traverses a shallow model and strips off one layer of modalities in every step. Alternatively, our algorithm may be seen as computing a shallow proof that enjoys a number of pleasant proof-theoretic properties, including a weak subformula property (i.e. it mentions only propositional combinations of subformulas of the goal).

The model construction relies on extending the axiomatisation of a given logic to a set of rules which is closed under rule resolution, i.e. every resolvent of two substituted rule conclusions can also be derived directly using a third rule. This process typically results in an infinite but recursive set of rules. Resolution closedness then enables us to build the shallow model using induction on the modal depth of formulas. Since we are working with an infinite set of rules, we have to impose a second condition to ensure that we can decide satisfiability: a rule set is closed under contraction if every substituted rule conclusion with duplicate literals can be derived using a substitution instance of a second rule in whose conclusion all literals remain distinct. The decision procedure will run in PSPACE if both closure under resolution and closure under contraction can be controlled, i.e. there is a polynomial bound on the size of rules that are applicable at every step of the deductive process. This turns out to be the case for all examples mentioned above. 
The material is organised as follows. In Section 2, we give a brief introduction to the generic coalgebraic semantics of modal logic. In Section 3, we discuss deduction systems for coalgebraic modal logics and their properties, notably the (equivalent) central notions of strict one-step completeness and reduction closedness of rule sets. Sections 4 and 5 are devoted to the tableau-based shallow model construction and the proof-theoretic view thereof. The ensuing PSPACE-algorithm and its example applications are presented in Section 6.

\section{COALGEBRAIC MODAL LOGIC}

We briefly recapitulate the basics of the coalgebraic interpretation of modal logic. To begin, we fix the syntactic framework. A modal signature is just a set $\Lambda$ of unary modal operators (all our results generalise straightforwardly to a polyadic setting as in [Schröder 2005]). The signature $\Lambda$ induces a modal language $\mathcal{F}(\Lambda)$, with formulas $\phi, \psi \in \mathcal{F}(\Lambda)$ defined by the grammar

$$
\phi::=\perp|\phi \wedge \psi| \neg \phi \mid L \phi,
$$

where $L$ ranges over $\Lambda$. Disjunctions $\phi \vee \psi$, truth $\top$, and other boolean operations are defined as usual. The depth of a formula is its maximal nesting depth of modal operators.

We work in the framework of coalgebraic modal logic, introduced by Pattinson [2004], generalising previous results [Jacobs 2000; Rößiger 2000; Kurz 2001; Pattinson 2001], where modal languages are interpreted over coalgebras for a Set-functor:

Definition 2.1. [Rutten 2000] Let $T:$ Set $\rightarrow$ Set be a functor, referred to as the signature functor, where Set is the category of sets. A $T$-coalgebra $A=(X, \xi)$ is a pair $(X, \xi)$ where $X$ is a set (of states) and $\xi: X \rightarrow T X$ is a function called the transition function. A morphism $f: A \rightarrow B$ between $T$-coalgebras $A=(X, \xi)$ and $B=(Y, \zeta)$ is a map $f: X \rightarrow Y$ such that $T f \xi=\zeta f$.

We view coalgebras as generalised transition systems: the transition function delivers a structured set of successors and observations for a state. Mutatis mutandis, we can in fact allow $T$ to take proper classes as values, as we never iterate $T$ or otherwise assume that $T X$ is a set; details are left implicit. This allows us to treat more examples, in particular Pauly's coalition logic (Example 2.7.8 below).

Assumption 2.2. We can assume w.l.o.g. that $T$ preserves injective maps [Barr 1993]. For convenience of notation, we will in fact sometimes assume that $T X \subseteq T Y$ in case $X \subseteq Y$. Moreover, we assume w.l.o.g. that $T$ is non-trivial, i.e. $T X=\emptyset \Longrightarrow$ $X=\emptyset$ (otherwise, $T X=\emptyset$ for all $X$ ).

Definition 2.3. If for a subset $Z \subseteq X$ of a coalgebra $A=(X, \xi), \xi$ restricts to a map $\xi_{Z}: Z \rightarrow T Z$, then $C=\left(Z, \xi_{Z}\right)$ is a subcoalgbra of $A$; in this case, the inclusion $Z \hookrightarrow X$ is a morphism $C \rightarrow A$.

In the same way that the signature functor abstracts from a concrete class of models, the interpretation of modal operators is encapsulated in terms of predicate liftings:

Definition 2.4. A predicate lifting for a functor $T$ is a natural transformation

$$
\mathcal{Q} \rightarrow \mathcal{Q} \circ T^{o p},
$$


where $\mathcal{Q}$ denotes the contravariant powerset functor Set $^{o p} \rightarrow$ Set (i.e. $\mathcal{Q}(X)=$ $\mathcal{P}(X)$ is the powerset, and $\mathcal{Q} f(B)=f^{-1}[B]$ for $f: X \rightarrow Y$ and $\left.B \in \mathcal{Q}(X)\right)$.

A coalgebraic semantics for a modal signature $\Lambda$ is given by a $\Lambda$-structure, consisting of a signature functor $T$ and an assignment of a predicate lifting $\llbracket L \rrbracket$ for $T$ to every modal operator $L \in \Lambda$; by abuse of notation, we refer to the entire $\Lambda$-structure just as $T$. Given a $\Lambda$-structure $T$, the satisfaction relation $\models_{C}$ between states $x$ of a $T$-coalgebra $C=(X, \xi)$ and $\mathcal{F}(\Lambda)$-formulas is defined inductively, with the usual clauses for the boolean operations. The clause for the modal operator $L$ is

$$
x \models_{C} L \phi \Longleftrightarrow \xi(x) \in \llbracket L \rrbracket_{C}\left(\llbracket \phi \rrbracket_{C}\right),
$$

where $\llbracket \phi \rrbracket_{C}=\left\{x \in X \mid x \models_{C} \phi\right\}$. We drop the subscripts $C$ when these are clear from the context.

We occasionally make use of the fact that the $\operatorname{logic} \mathcal{F}(\Lambda)$ is adequate for $T$ coalgebras [Pattinson 2004]:

Proposition 2.5. If $f: A \rightarrow B$ is a morphism of $T$-coalgebras, then

$$
x \models_{A} \phi \quad \text { iff } \quad f(x) \models_{B} \phi
$$

for all states $x$ in $A$ and all $\mathcal{F}(\Lambda)$-formulas $\phi$.

Our main interest here is in the local satisfiability problem:

Definition 2.6. An $\mathcal{F}(\Lambda)$-formula $\phi$ is satisfiable (over $T$ ) if there exist a $T$ coalgebra $A=(X, \xi)$ and a state $x$ in $X$ such that $x \models_{A} \phi$. Dually, $\phi$ is valid if $x \models_{A} \phi$ for all $T$-coalgebras $A=(X, \xi)$ and all $x \in X$.

Example 2.7. [Pattinson 2004; Cîrstea and Pattinson 2007; Schröder 2007] We illustrate how the coalgebraic approach subsumes a large class of modal logics. This includes not only logics with a standard Kripke semantics, but in particular also non-normal modal logics whose semantics is defined over structures that differ substantially from classical Kripke frames.

(1) Modal logic $K$ : The signature $\Lambda_{K}$ of the modal logic $K$ consists of a single modal operator $\square$. Let $\mathcal{P}$ be the covariant powerset functor. Then $\mathcal{P}$-coalgebras are graphs, thought of as transition systems or indeed Kripke frames. A $\Lambda_{K}$-structure over $\mathcal{P}$ is defined by

$$
\llbracket \square \rrbracket_{X}(A)=\{B \in \mathcal{P}(X) \mid B \subseteq A\} ;
$$

this induces precisely the standard Kripke semantics of modal logic (note that no restrictions are imposed on frames).

(2) Modal logic $K D$ : $K D$ is obtained from $K$ by adding the axiom $\neg \square \perp$, i.e. by restricting the semantics to serial Kripke frames $(X, R)$, characterized by the condition that for every state $x$, there exists a state $y$ such that $x R y$. Thus, the signature $\Lambda_{K D}$ of the normal modal logic $K D$ is the same as that of $K$, and a $\Lambda_{K D^{-}}$ structure is defined in the same way as for $K$, but over the non-empty powerset functor $\mathcal{P}^{*}$ defined by $\mathcal{P}^{*}(X)=\{A \in \mathcal{P}(X) \mid A \neq \emptyset\}$.

(3) Modal logic $E$ : The signature $\Lambda_{E}$ of the modal logic $E$, the smallest classical modal logic [Chellas 1980], has a single modal operator $\square$; the proof system of $E$ comprises, besides propositional reasoning, only replacement of equivalents (i.e. 
the rule $a \leftrightarrow b / \square a \rightarrow \square b)$. The standard neighbourhood semantics of $E$ is coalgebraically captured by a $\Lambda_{E}$-structure over the neighbourhood functor $N=\mathcal{Q} \circ \mathcal{Q}^{o p}$ (composition of the contravariant powerset functor with itself); coalgebras for this functor are neighbourhood frames. The modal operator $\square$ is interpreted over $N$ by

$$
\llbracket \square \rrbracket_{X}(A)=\{\alpha \in N(X) \mid A \in \alpha\} .
$$

(4) Modal logic $M$ : The modal logic $M$, the smallest monotonic modal logic [Chellas 1980], is obtained from the modal logic $E$ by adding the monotonicity rule $a \rightarrow b / \square a \rightarrow \square b$. The neighbourhood semantics of $M$ is captured coalgebraically analogously to the previous example as a structure over the subfunctor Up $\mathcal{P}$ of $N$ assigning to a set $X$ the set of upwards closed subsets of $\mathcal{Q} X$. Coalgebras for UpP are monotone neighbourhood frames [Hansen and Kupke 2004].

(5) Graded modal logic [Fine 1972]: The modal signature of graded modal logic (GML) is $\Lambda_{G M L}=\left\{\diamond_{k} \mid k \in \mathbb{N}\right\}$; the intended reading of $\diamond_{k} \phi$ is ' $\phi$ holds in more than $k$ successor states'. The semantics of GML is originally defined by counting successor states in Kripke frames. This semantics fails to be coalgebraic, as the naturality condition for the associated predicate liftings fails. However, one may define a coalgebraic semantics which is equivalent for purposes of satisfiability [Schröder 2007], as follows. The finite multiset (or bag) functor $\mathcal{B}$ maps a set $X$ to the set of maps $B: X \rightarrow \mathbb{N}$ with finite support, the intuition being that $B$ is a multiset containing $x \in X$ with multiplicity $B(x)$. We extend $B$ to $\mathcal{P}(X)$ by putting $B(A)=\sum_{x \in A} B(x)$. The action on morphisms $f: X \rightarrow Y$ is then given by $\mathcal{B} f: \mathcal{B} X \rightarrow \mathcal{B} Y, B \mapsto \lambda y . B\left(f^{-1}[\{y\}]\right)$. Coalgebras for $\mathcal{B}$ are directed graphs with $\mathbb{N}$-weighted edges, often referred to as multigraphs [D'Agostino and Visser 2002]. The graded modal operator $\diamond_{k}$ is intepreted over $\mathcal{B}$ by

$$
\llbracket \diamond_{k} \rrbracket_{X}(A)=\{B: X \rightarrow \mathbb{N} \in \mathcal{B}(X) \mid B(A)>k\} .
$$

Thus, $x \vDash \diamond_{k} \phi$ for a state $x$ in a $\mathcal{B}$-coalgebra iff $\phi$ holds for more than $k$ successor states of $x$, taking into account multiplicities.

The dual operators $\neg \diamond_{k} \neg$ are denoted $\square_{k}$, i.e. $\square_{k} \phi$ reads ' $\phi$ fails in at most $k$ successor states'. Note that $\square_{k}$ is monotone, but fails to be normal unless $k=0$. A non-monotone variation of GML arises when negative multiplicities are admitted.

(6) Majority logic [Pacuit and Salame 2004]: Graded modal logic is extended to majority logic by adding a weak majority operator $W$, read 'in at least half of the successor states, it is the case that ...' . The structure for GML over the multiset functor $\mathcal{B}$ described in the previous example is extended to $W$ by putting

$$
\llbracket W \rrbracket_{X}(A)=\{B: X \rightarrow \mathbb{N} \in \mathcal{B}(X) \mid B(A) \geq B(X-A)\} .
$$

The dual operator $M=\neg W \neg$ captures strict majority 'in more than half of the successor states, it is the case that'.

(7) Probabilistic modal logic [Larsen and Skou 1991; Heifetz and Mongin 2001]: The modal signature $\Lambda_{P M L}$ of probabilistic modal logic (PML) comprises operators $L_{p}, p \in[0,1] \cap \mathbb{Q}$, to be read 'in the next step, it is with probability at least $p$ the case that...'. We define a $\Lambda_{P M L}$-structure over the finite distribution functor $D_{\omega}$ which maps a set $X$ to the set of probability distributions on $X$ with finite 
support. Coalgebras for $D_{\omega}$ are probabilistic transition systems (also called probabilistic type spaces [Heifetz and Mongin 2001]) with finite branching degree. Our definition contrasts with that of [Heifetz and Mongin 2001], where there is no restriction on the branching degree, but since PML has the finite model property (cf. loc. cit.), this has no bearing on satisfiability. The interpretation of $L_{p}$ over $D_{\omega}$ is defined by

$$
\llbracket L_{p} \rrbracket(A)=\left\{P \in D_{\omega} X \mid P A \geq p\right\} .
$$

PML is non-normal $\left(L_{p}(a \vee b) \rightarrow L_{p} a \vee L_{p} b\right.$ is not valid for $\left.p>0\right)$.

(8) Coalition logic [Pauly 2002]: Let $N=\{1, \ldots, n\}$ be a fixed set of agents. Subsets of $N$ are called coalitions. The signature $\Lambda_{\text {Coal }}$ of coalition logic consists of modal operators $[C]$, where $C$ ranges over coalitions, read 'coalition $C$ has a collaborative strategy to ensure that ...'. A coalgebraic semantics for coalition logic is based on the class-valued signature functor $T$ defined by

$$
T X=\left\{\left(S_{1}, \ldots, S_{n}, f\right) \mid \emptyset \neq S_{i} \in \text { Set }, f: \prod_{i \in N} S_{i} \rightarrow X\right\} .
$$

The elements of $T X$ are understood as strategic games with set $X$ of states, i.e. tuples consisting of nonempty sets $S_{i}$ of strategies for all agents $i$, and an outcome function $\left(\prod S_{i}\right) \rightarrow X$. A $T$-coalgebra is a game frame [Pauly 2002]. We denote the set $\prod_{i \in C} S_{i}$ by $S_{C}$, and for $\sigma_{C} \in S_{C}, \sigma_{\bar{C}} \in S_{\bar{C}}$, where $\bar{C}=N-C,\left(\sigma_{C}, \sigma_{\bar{C}}\right)$ denotes the obvious element of $\prod_{i \in N} S_{i}$. A $\Lambda_{C o a l}$-structure over $T$ is then defined by

$$
\llbracket[C] \rrbracket_{X}(A)=\left\{\left(S_{1}, \ldots, S_{n}, f\right) \in T X \mid \exists \sigma_{C} \in S_{C} \cdot \forall \sigma_{\bar{C}} \in S_{\bar{C}} \cdot f\left(\sigma_{C}, \sigma_{\bar{C}}\right) \in A\right\} .
$$

All the above examples can be canonically extended to systems that process inputs from a set $I$ by passing from the signature functor $T$ to one of the functors $T^{I}$ or $T\left(I \times \times_{-}\right)$and suitably indexing the modal operators. We refer to [Cîrstea and Pattinson 2007] for a detailed account of the induced logics.

Remark 2.8. In the modal grammar given above, atomic propositional symbols are deliberately not included. This is for the sake of both generality, as some modal logics such as Hennessy-Milner logic do not include such atomic propositions, and economy of presentation, as a set $U$ of atomic propositional symbols may be integrated in the basic framework as follows. Given a modal signature $\Lambda$ and a $\Lambda$-structure $T$, we define a structure for the modal signature $\Lambda_{U}=\Lambda \cup U$ over the functor $T_{U}$ defined by $T_{U} X=T X \times \mathcal{P}(U)$ : modal operators from $\Lambda$ are interpreted by taking the preimage of their interpretation over $T$ under the projection $T_{U} \rightarrow T$, and a propositional symbol $a \in U$ is interpreted by putting

$$
\llbracket a \rrbracket_{X}(A)=\{(t, B) \in T X \times \mathcal{P}(U) \mid a \in B\} .
$$

Since $\llbracket a \rrbracket$ is independent of its argument, the modal operator $a$ can be written as just the propositional symbol $a$ (without an argument formula). In a framework with polyadic modal operators [Schröder 2005], propositional constants correspond to nullary modalities. Some of the logics above indeed require propositional symbols lest they collapse into triviality. This holds in those cases where $T 1$ (for 1 a singleton set) is a singleton, e.g. probabilistic modal logic, coalition logic, and the modal logic $K D$. We nevertheless generally continue to omit the treatment of propositional symbols in the sequel, since the addition of propositional symbols as indicated above 
has no bearing on the rule sets forming the core of our method, and the model construction is entirely analogous.

\section{PROOF SYSTEMS FOR COALGEBRAIC MODAL LOGIC}

Our decision procedure for rank- 1 logics relies on a complete axiomatisation in a certain format. Deduction for modal logics with coalgebraic semantics has been considered in [Pattinson 2003; Cîrstea and Pattinson 2007; Kupke et al. 2005; Schröder 2007]. It has been shown that every modal logic over coalgebras can be axiomatised in rank 1 using either rank-1 axioms or rules leading from rank 0 to rank 1 [Schröder 2007], essentially because functors, as opposed to comonads, only encode the one-step behaviour of systems. Here, we focus on rules. The crucial ingredients for the shallow model construction and the ensuing PSPACE algorithm are novel notions of resolution closure and strict one-step completeness of rule sets.

For the remainder of the paper, we fix a modal signature $\Lambda$ and a $\Lambda$-structure $T$. We recall a few basic notions from propositional logic, as well as notation for coalgebraic modal logic introduced in [Pattinson 2003; Cîrstea and Pattinson 2007]:

Definition 3.1. We denote the set of propositional formulas over a set $V$ (consisting e.g. of propositional variables or modal formulas) by $\operatorname{Prop}(V)$. Here, we regard $\neg$ and $\wedge$ as the basic connectives, with all other connectives defined in the standard way. For $\phi, \psi \in \operatorname{Prop}(V)$, we say that $\phi$ propositionally entails $\psi$ and write $\phi \vdash_{P L} \psi$ if $\phi \rightarrow \psi$ is a propositional tautology. Similarly, $\Phi \subseteq \operatorname{Prop}(V)$ propositionally entails $\psi\left(\Phi \vdash_{P L} \psi\right)$ if there exist $\phi_{1}, \ldots, \phi_{n} \in \Phi$ such that $\phi_{1} \wedge \cdots \wedge \phi_{n} \vdash_{P L} \psi$.

A literal over $V$ is either an element of $V$ or the negation of such an element. We use the meta-variable $\epsilon$ (possibly indexed) to denote either nothing or $\neg$, so that a literal over $V$ has the general form $\epsilon a, a \in V$. A clause is a finite (possibly empty) disjunction of literals, which then takes the form $\bigvee_{i=1}^{n} \epsilon_{i} a_{i}$ with $a_{1}, \ldots, a_{n} \in V$. Similarly, a conjunctive clause is a finite conjunction of literals. A (conjunctive) clause is contracted if all its literals are distinct. The set of all clauses over $V$ is denoted by $\mathrm{Cl}(V)$. Although we regard clauses as formulas rather than sets of literals, we shall sometimes use terminology such as 'a literal is contained in a clause' or 'a clause contains another', with the obvious meaning. We denote by $\mathrm{Up}(V)$ the set $\{L a \mid L \in \Lambda, a \in V\}$.

If $V$ consists of propositional variables, then we have the usual notions of valuation and substitution: A valuation is just a map $\kappa: V \rightarrow\{\top, \perp\}$ assigning boolean truth values to variables; for $\phi \in \operatorname{Prop}(V)$, we write $\kappa \models \phi$ if $\kappa$ is a satisfying valuation for $\phi$. More generally, given a set $X$, a $\mathcal{P}(X)$-valuation for $V$ is a map $V \rightarrow \mathcal{P}(X)$. For $\phi \in \operatorname{Prop}(V)$, a $\mathcal{P}(X)$-valuation $\tau$ induces in the obvious way a subset $\llbracket \phi \rrbracket \tau$ of $X$; we write $X, \tau \models \phi$ if $\llbracket \phi \rrbracket \tau=X$. Using the structure $T$ for $\Lambda$, we interpret $\psi \in \operatorname{Prop}(\operatorname{Up}(V))$ as a subset $\llbracket \psi \rrbracket \tau$ of $T X$ by putting $\llbracket L \phi \rrbracket \tau=\llbracket L \rrbracket \llbracket \phi \rrbracket \tau$, and we write $T X, \tau \models \psi$ if $\llbracket \psi \rrbracket \tau=T X$. Moreover, given a set $Z$, a $Z$-substitution for $V$ is a map $\sigma: V \rightarrow Z$; for a formula $\phi$ over $V($ e.g. $\phi \in \operatorname{Prop}(\operatorname{Up}(\operatorname{Prop}(V))))$, we denote the result of performing the substitution $\sigma$ on $\phi$ by $\phi \sigma$ and refer to $\phi \sigma$ as a $Z$-instance of $\phi$.

Lemma 3.2. For $\phi, \psi \in \mathrm{Cl}(V), \phi \vdash_{P L} \psi$ iff either $\phi$ is contained in $\psi$ or $\psi$ is a tautology (i.e. contains both $a$ and $\neg$ a for some $a \in V$ ). 
Definition 3.3. A (one-step) rule $R$ over a set $V$ of propositional variables is a rule $\phi / \psi$, where $\phi \in \operatorname{Prop}(V)$ and $\psi \in \mathrm{Cl}(\operatorname{Up}(V))$. We silently identify rules modulo $\alpha$-equivalence. The rule $R$ is sound if, whenever $\phi \sigma$ is valid for an $\mathcal{F}(\Lambda)$ substitution $\sigma$, then $\psi \sigma$ is valid. Moreover, $R$ is one-step sound if $T X, \tau \models \psi$ for each set $X$ and each $\mathcal{P}(X)$-valuation $\tau$ such that $X, \tau \models \phi$.

Our hitherto informal use of the term rank-1 logic formally means axiomatisable by one-step rules. The term rank-1 logic has been used in the literature [Pattinson 2003; Cîrstea and Pattinson 2007; Kupke et al. 2005; Schröder 2007] to describe logics axiomatisable by rank-1 axioms, i.e. propositional combinations of formulas $L \phi$ where $L$ is a modal operator and $\phi$ is purely propositional (in the notation introduced above, formulas from $\operatorname{Prop}(\operatorname{Up}(\operatorname{Prop}(V))))$. This class of axioms includes e.g. the $K$ axiom $\square(a \rightarrow b) \rightarrow(\square a \rightarrow \square b)$, but excludes axioms containing nested modalities or top-level propositional variables such as the axioms 4 and $T$, respectively. It has been shown in [Schröder 2007] that one-step rules and rank-1 axioms determine the same class of logics.

Remark 3.4. We can always assume that every propositional variable $a$ appearing in the premise $\phi$ of a one-step rule appears also in the conclusion: otherwise, we can eliminate $a$ by passing from $\phi$ to $\phi[\top / a] \vee \phi[\perp / a]$.

Proposition 3.5. [Schröder 2007] Every one-step sound rule is sound.

The converse holds under additional assumptions [Schröder 2005]; note however that the obviously sound rule $\perp / \perp$ is one-step sound iff $T \emptyset=\emptyset$ (as is the case e.g. for PML).

A given set $\mathcal{R}$ of one-step sound rules induces a proof system for $\mathcal{F}(\Lambda)$ as follows.

Definition 3.6. Let $\mathcal{R}_{C}$ denote the set of rules obtained by extending $\mathcal{R}$ with the congruence rule

$$
\text { (C) } \frac{a \leftrightarrow b}{L a \rightarrow L b}
$$

for every $L \in \Lambda$. (This rule of course implies a rule where $\rightarrow$ is replaced by $\leftrightarrow$, which however does not fit the format for one-step rules.) The set of provable formulas is the smallest set closed under propositional entailment and the rules in $\mathcal{R}_{C}$, with propositional variables instantiated to formulas in $\mathcal{F}(\Lambda)$. We say that a formula $\phi$ is consistent if $\neg \phi$ is not provable.

It is easy to see that this proof system is sound. Completeness requires 'enough' rules in the following sense.

Definition 3.7. The set $\mathcal{R}$ is (strictly) one-step complete if, whenever $T X, \tau \models \chi$ for a set $X, \chi \in \mathrm{Cl}(\mathrm{Up}(V))$, and a $\mathcal{P}(X)$-valuation $\tau$, then $\chi$ is (strictly) provable over $X, \tau$, i.e. propositionally entailed by clauses (a clause) $\psi \sigma$ where $\phi / \psi \in \mathcal{R}_{C}$ (Definition 3.6) and $\sigma$ is a $\operatorname{Prop}(V)$-substitution (a $V$-substitution) such that $X, \tau \models$ $\phi \sigma$.

Strict one-step completeness is one of crucial notions in this work. Its distinctive feature is that strict provability largely dispenses with propositional reasoning by restricting instantiations to propositional variables, and by replacing general 
propositional entailment by the rather trivial concept of propositional entailment between single clauses (cf. Lemma 3.2). This plays a central role in the shallow model construction presented in Section 4.

Remark 3.8. It is shown in [Schröder 2007] that the set of all one-step sound rules is always strictly one-step complete and that the proof system induced by a one-step complete set of rules is weakly complete, i.e. proves all valid formulas.

In the further treatment, we need a further technical condition.

Definition 3.9. A one-step rule $\phi / \psi$ over $V$ is injective if every variable in $V$ occurs at most once in $\psi$.

Assumption 3.10. We assume for the remainder of the paper that the given rules in $\mathcal{R}$ are injective. This restriction will be satisfied by the naturally arising rule sets in our examples; it can always be forced by introducing new propositional variables and adding premises stating the equivalence to the original variables (e.g. a rule $\top /(\square a \rightarrow \diamond a)$ can be replaced by $(a \leftrightarrow b) /(\square a \rightarrow \diamond b))$.

Strictly one-step complete sets of rules are generally more complicated than onestep complete sets of rules or axioms [Pattinson 2003; Schröder 2007]. In our terminology, part of the effort of [Vardi 1989] and [Pauly 2002] is devoted to finding strictly one-step complete sets of rules. We now develop a systematic procedure for turning one-step complete rule sets into strictly one-step complete ones. For the following, recall that given clauses $\phi$ and $\psi$ containing literals $a$ and $\neg a$, respectively, a resolvent of $\phi$ and $\psi$ (at $a$ ) is obtained by removing $a$ and $\neg a$ from the clause $\phi \vee \psi$. A set $\Phi$ of clauses is called resolution closed if, for $\phi, \psi \in \Phi$, all resolvents of $\phi$ and $\psi$ are propositionally entailed by some clause in $\Phi$. This is generalised to rules as follows:

Definition 3.11. A set $\mathcal{R}$ of one-step rules is resolution closed if it satisfies the following requirement. Let $R_{1}, R_{2} \in \mathcal{R}$, where $R_{1}=\phi_{1} / \psi_{1}$ and $R_{2}=\phi_{2} / \psi_{2}$. We can assume that $R_{1}$ and $R_{2}$ have disjoint sets $V_{1}, V_{2}$ of propositional variables. Let $L a$ be in $\psi_{1}$, and let $\neg L b$ be in $\psi_{2}$ for some $L \in \Lambda$, so that we have a resolvent $\bar{\psi}$ of $\psi_{1}$ and $\psi_{2}[a / b]$ at $L a$; by Assumption 3.10, $\bar{\psi}$ is a clause over $\operatorname{Up}(V)$ where $V=V_{1} \cup V_{2}-\{a, b\}$. Then $\mathcal{R}_{C}$ is required to contain a rule $R=\phi / \psi$ such $\phi_{1} \wedge \phi_{2}[a / b] \vdash_{P L} \phi \sigma$ and $\psi \sigma \vdash_{P L} \bar{\psi}$ for some $V$-substitution $\sigma$; in this case, $R$ is called a resolvent of $R_{1}$ and $R_{2}$.

Resolution closure will play a central role in the following development, as it forms the syntactic counterpart of strict one-step completeness.

Remark 3.12. One can construct resolution closed sets by iterated addition of missing resolvents. Here, an obvious choice for a resolvent $\phi / \psi$ of $\phi_{1} / \psi_{1}$ and $\phi_{2} / \psi_{2}$ as above is to take $\psi$ as the resolvent $\bar{\psi}$ of $\psi_{1}$ and $\psi_{2}$, and $\phi$ as $\phi_{1} \wedge \phi_{2}[a / b]$, with $a$ eliminated according to Remark 3.4 as $a$ is not contained in $\psi$ by Assumption 3.10. It is clear that $\phi_{1} \wedge \phi_{2}[a / b] / \bar{\psi}$ is one-step sound if $R_{1}$ and $R_{2}$ are one-step sound.

Remark 3.13. Note that our approach is different to existing resolution-based approaches to decision procedures for modal logic (e.g. [De Nivelle et al. 2000]), which rely on translating modal logic into first-order logic. 
Lemma 3.14. Let $\psi \in \mathrm{Cl}(V)$, and let $\emptyset \neq \Phi \subseteq \mathrm{Cl}(V)$ be resolution closed. Then $\Phi \vdash_{P L} \psi$ iff $\phi \vdash_{P L} \psi$ for some $\phi \in \Phi$.

Proof. The 'if' direction is clear. 'Only if': W.l.o.g. $\psi$ is not a tautology. We can assume that $V$ is finite and then prove the contraposition of the claim by induction over the size of $V$. Thus assume, recalling Lemma 3.2, that $\Phi$ does not contain a subclause of $\psi$. Pick a clause $\chi \in \Phi$ that contains a minimal number of literals not in $\psi$ (this number is non-zero); w.l.o.g. $\chi$ contains a positive literal $a$ such that $a$ is not in $\psi$. Remove all clauses containing $a$ from $\Phi$, and remove $\neg a$ from the remaining clauses and from $\psi$, obtaining a new set $\Phi^{\prime}$ of clauses and a new clause $\psi^{\prime}$, respectively. Then $\Phi^{\prime}$ is resolution closed and does not contain a subclause of $\psi^{\prime}$ (otherwise there exists a clause $\rho \in \Phi$ whose only literal not in $\psi$ is $\neg a$, and resolving $\rho$ with $\chi$ yields a clause in $\Phi$ with less literals not in $\psi$ than $\chi$, contradiction). By induction we thus have a valuation $\tau^{\prime}$ for $V-\{a\}$ satisfying $\Phi^{\prime}$ but not $\psi^{\prime}$. We extend $\tau^{\prime}$ to a valuation $\tau$ for $V$ by putting $\tau(a)=\mathrm{T}$; then $\tau$ satisfies $\Phi$ but not $\psi$.

LEMMA 3.15. $\mathcal{R}$ is resolution closed iff $\mathcal{R}_{C}$ is resolution closed.

Proof. The 'if' direction is trivial. The 'only if' direction follows from the fact that every rule $R$ is a resolvent of $R$ and any congruence rule, since rules are injective (Assumption 3.10).

TheOREm 3.16. Let $\mathcal{R}$ be one-step complete. Then $\mathcal{R}$ is strictly one-step complete iff $\mathcal{R}$ is resolution closed.

Proof. 'If': Let $X$ be a set, let $\tau$ be a $\mathcal{P}(X)$-valuation, and let $\chi \in \mathrm{Cl}(\mathrm{Up}(V))$ such that $T X, \tau \models \chi$; w.l.o.g. $\chi$ is not a tautology. By one-step completeness, $\chi$ is propositionally entailed by the (non-empty) set of clauses

$$
\Psi=\left\{\psi \sigma \mid \phi / \psi \in \mathcal{R}_{C}, \sigma \text { a } \operatorname{Prop}(V) \text {-substitution, } X, \tau \models \phi \sigma\right\} .
$$

The set $\Psi$ is resolution closed: for $i=1,2$, let $\phi_{i} / \psi_{i} \in \mathcal{R}_{C}$ be a rule over $W_{i}$ (with $W_{1}, W_{2}$ disjoint), let $\sigma_{i}$ be a $\operatorname{Prop}(V)$-substitution such that $X, \tau \models \phi_{i} \sigma_{i}$, and let $\psi_{1} \sigma_{1}$ and $\psi_{2} \sigma_{2}$ contain literals $L \rho$ and $\neg L \rho$, respectively. Thus, $\psi_{1}$ and $\psi_{2}$ contain literals $L a$ and $\neg L b$, respectively, where $\sigma_{1}(a)=\sigma_{2}(b)=\rho$; let $\bar{\psi}$ be the resolvent of $\psi_{1}, \psi_{2}[a / b]$ at $L a$, a clause over $W=W_{1} \cup W_{2}-\{a, b\}$. Then the resolvent of $\psi_{1} \sigma_{1}, \psi_{2} \sigma_{2}$ at $L \rho$ is $\bar{\psi} \sigma$, where $\sigma$ acts like $\sigma_{1}$ on $W_{1}-\{a\}$ and like $\sigma_{2}$ on $W_{2}-\{b\}$. By resolution closedness of $\mathcal{R}_{C}$ (Lemma 3.15), we have $\phi / \psi \in \mathcal{R}_{C}$ and a $W$-substitution $\theta$ such that $\phi_{1} \wedge \phi_{2}[a / b] \vdash_{P L} \phi \theta$ and $\psi \theta \vdash_{P L} \bar{\psi}$. Then $X, \tau \models \phi \theta \sigma$, so that $\psi \theta \sigma \in \Psi$, and $\psi \theta \sigma \vdash_{P L} \bar{\psi} \sigma$ as required.

By Lemma 3.14, it now follows that $\psi \sigma \vdash_{P L} \chi$ for some clause $\psi \sigma$ in $\Psi$, where by Lemma 3.2 necessarily $\sigma(v) \in V$ for every variable $v$ in $\psi$.

'Only if': Let $\phi_{1} / \psi_{2}, \phi_{2} / \psi_{2} \in \mathcal{R}$ be rules over disjoint sets $V_{1}, V_{2}$ of variables, where $\psi_{1}$ contains $L a$ and $\psi_{2}$ contains $\neg L b$. Let $\bar{\psi}$ denote the resolvent of $\psi_{1}, \psi_{2}[a / b]$ at $L a$, a clause over $V=V_{1} \cup V_{2}-\{a, b\}$. Let $X$ be the set of satisfying valuations for $\phi_{1} \wedge \phi_{2}[a / b]$, and define the $\mathcal{P}(X)$-valuation $\tau$ by $\tau(a)=\{\kappa \in X \mid \kappa(a)=\top\}$. Then $X, \tau \models \phi_{1} \wedge \phi_{2}[a / b]$ and hence $T X, \tau \models \bar{\psi}$ by one-step soundness of $\mathcal{R}$. By strict one-step completeness, it follows that there exists a rule $\phi / \psi \in \mathcal{R}_{C}$ and a $V$-substitution $\sigma$ such that $X, \tau \models \phi \sigma$ and $\psi \sigma \vdash_{P L} \bar{\psi}$. By construction of $X, \tau$, we may conclude from $X, \tau \models \phi \sigma$ that $\phi_{1} \wedge \phi_{2}[a / b] \vdash_{P L} \phi \sigma$ as required. 
In summary, strictly one-step complete rule sets can be constructed by resolving the rules of a one-step complete axiomatisation against each other. Below, we give examples of strictly one-step complete systems obtained in this way. In order to simplify the presentation for the case of graded modal logic and probabilistic modal logic, we use the following notation. If $\phi_{i}$ is a formula, $r_{i} \in \mathbb{Z}$ for all $i \in I$, and $k \in \mathbb{Z}$, we abbreviate

$$
\sum_{i \in I} r_{i} \phi_{i} \geq k \equiv \bigwedge_{\substack{J \subseteq I \\ r(J)<k}}\left(\bigwedge_{j \in J} \phi_{j} \rightarrow \bigvee_{j \notin J} \phi_{j}\right),
$$

where $r(J)=\sum_{j \in J} r_{j}$. The formula $\sum_{i \in I} r_{i} a_{i} \geq k$ translates into the arithmetic of characteristic functions as suggested by the notation:

Lemma 3.17. An element $x \in X$ belongs to the interpretation of $\sum_{i \in I} r_{i} a_{i} \geq k$ under a $\mathcal{P}(X)$-valuation $\sigma$ iff

$$
\sum_{i \in I} r_{i} \mathbb{1}_{\sigma\left(a_{i}\right)}(x) \geq k
$$

where $\mathbb{1}_{A}: X \rightarrow\{0,1\}$ is the characteristic function of $A \subseteq X$.

Proof. The element $x$ satisfies the negation of $\sum_{i \in I} r_{i} a_{i} \geq k$ iff $r(J)<k$ for $J=\left\{i \in I \mid x \in \sigma\left(a_{i}\right)\right\}$ iff $\sum_{i \in I} r_{i} \mathbb{1}_{\sigma\left(a_{i}\right)}(x)<k$.

We allow ourselves obvious variations of this notation, e.g. $\sum a_{i} \leq \sum b_{j}$ in place of $\sum b_{j}-\sum a_{i} \geq 0$.

In all the logics of Example 2.7, the resolution process, applied to known one-step complete rule sets, can be kept under control; by Theorem 3.16, the resulting rule sets are strictly one-step complete.

Example 3.18. (1) Modal logic E: The empty set of rules is one-step complete for neighbourhood frame semantics (Example 2.7.3). This set is trivially resolution closed.

(2) Modal logic $M$ : The one-step rule

$$
(M) \frac{a \rightarrow b}{\square a \rightarrow \square b}
$$

is one-step complete for monotone neighbourhood frame semantics (Example 2.7.3), and clearly resolution closed.

(3) Modal logic K: The one-step rules

$$
\frac{a}{\square a} \quad \frac{a \wedge b \rightarrow c}{\square a \wedge \square b \rightarrow \square c}
$$

are one-step complete for unrestricted Kripke semantics (Example 2.7.1), i.e. for the modal logic $K$ [Pattinson 2003]. The resolution closure $\mathcal{R}$ of these rules consists of the rules

$$
\frac{\bigwedge_{i=1}^{n} a_{i} \rightarrow b}{\bigwedge_{i=1}^{n} \square a_{i} \rightarrow \square b}
$$

for all $n \in \mathbb{N}$ (here, strict one-step completeness is also easily seen directly). Note the similarity between this rule and a corresponding rule appearing in standard 
cut-free sequent calculi for $K$ [Troelstra and Schwichtenberg 1996]; the precise connection between resolution closure and cut elimination is the subject of further investigation.

(4) Modal logic KD: The axiomatisation of $K$ is extended to a one-step complete axiomatisation of $K D$ (Example 2.7.2) by adding the rule $\neg a / \neg \square a$. Closing the new rule set under resolution leads to the rules

$$
\frac{\bigwedge_{i=1}^{n} a_{i} \rightarrow b}{\bigwedge_{i=1}^{n} \square a_{i} \rightarrow \square b} \text { and } \frac{\neg \bigwedge_{i=1}^{n} a_{i}}{\neg \bigwedge_{i=1}^{n} \square a_{i}}
$$

for all $n \in \mathbb{N}$ (i.e. where the rules of $K$ apply only to positive Horn clauses, the rules of $K D$ apply to arbitrary Horn clauses).

(5) Coalition logic: In Lemma 6.1 of [Pauly 2002], the following set of one-step rules for coalition logic (Example 2.7.8), numbered as in loc. cit., is implicit:

$$
\begin{gathered}
\text { (1) } \frac{\bigvee_{i=1}^{n} \neg a_{i}}{\bigvee_{i=1}^{n} \neg\left[C_{i}\right] a_{i}} \quad \text { (2) } \frac{a}{[C] a} \quad \text { (3) } \frac{a \vee b}{[0] a \vee[N] b} \\
\text { (4) } \frac{\bigwedge_{i=1}^{n} a_{i} \rightarrow b}{\bigwedge_{i=1}^{n}\left[C_{i}\right] a_{i} \rightarrow\left[\bigcup C_{i}\right] b}
\end{gathered}
$$

where $n \geq 0$, and rules (1) and (4) are subject to the side condition that the $C_{i}$ are pairwise disjoint.

As shown in [Pauly 2002], an axiomatization subsumed by rules (1)-(4) is complete for a language including propositional symbols; one-step completeness follows by Proposition 5.3 below. The rules are moreover 'nearly' resolution closed (full resolution closure is not needed in [Pauly 2002] due to the use of a taylored notion of closed rule set). Resolving rule (4) with rules (2) and (3), one obtains the rule schema

$$
\left(4^{\prime}\right) \frac{\bigwedge_{i=1}^{n} a_{i} \rightarrow b \vee \bigvee_{j=1}^{m} c_{j}}{\bigwedge_{i=1}^{n}\left[C_{i}\right] a_{i} \rightarrow[D] b \vee \bigvee_{j=1}^{m}[N] c_{j}}
$$

where $m, n \geq 0$, subject to the side condition that the $C_{i}$ are pairwise disjoint subsets of $D$; this subsumes rules (2)-(4) above.

Resolution closedness of rules (1) and (4'): We discuss only the case of resolving $\left(4^{\prime}\right)$ against itself; the other case is similar. Let one instance of $\left(4^{\prime}\right)$ be denoted as in the rule schema, and another instance with all entities primed $\left(a_{i}^{\prime}\right.$ etc.). The two instances can be resolved in two essentially different ways. The subcase where matching is with $[D] b$ is straightforward. Thus assume w.l.o.g. that matching is via $[N] c_{1} \equiv\left[C_{1}^{\prime}\right] a_{1}^{\prime}$. Then by the side conditions, $D^{\prime}=N$ and $C_{i}^{\prime}=\emptyset$ for $i=2, \ldots, n^{\prime}$. Thus, the resolvent has the conclusion

$$
\bigwedge_{i=1}^{n}\left[C_{i}\right] a_{i} \wedge \bigwedge_{i=2}^{n^{\prime}}\left[C_{i}^{\prime}\right] a_{i}^{\prime} \rightarrow[D] b \vee[N] b^{\prime} \vee \bigvee_{j=2}^{m}[N] c_{j} \vee \bigvee_{j=1}^{m^{\prime}}[N] c_{j}^{\prime},
$$

which fits the format of the rule scheme $\left(4^{\prime}\right)$. It is easy to check that the combined premises imply the required premise for the resolved conclusion, and similarly for the side conditions.

(6) Graded modal logic: The standard axiomatization of graded modal logic, 
weakly complete for a language with propositional symbols [Caro 1988], has axioms

$$
\begin{aligned}
& \diamond_{n+1} a \rightarrow \diamond_{n} a \\
& \square_{0}(a \rightarrow b) \rightarrow \diamond_{n} a \rightarrow \diamond_{n} b \\
& \diamond !_{0}(a \wedge b) \rightarrow\left(\left(\diamond !_{n_{1}} a \wedge \diamond !_{n_{2}} b\right) \rightarrow \diamond !_{n_{1}+n_{2}}(a \vee b)\right)
\end{aligned}
$$

$\square_{0} \top$

where $n, n_{1}, n_{2} \in \mathbb{N}$, used in a proof system including propositional reasoning and the congruence rule (so that $(N)$ induces the necessition rule for $\square_{0}$ ). Here, $\diamond !_{n} \phi$ abbreviates $\diamond_{n-1} \phi \wedge \neg \diamond_{n} \phi$ for $n>0$, and $\neg \diamond_{0} \phi$ for $n=0$. These axioms may be derived from the system of one-step rules

$$
\begin{gathered}
(R G 1) \frac{a \rightarrow b}{\diamond_{n+1} a \rightarrow \diamond_{n} b} \quad(A 1) \frac{c \rightarrow a \vee b}{\diamond_{n_{1}+n_{2}} c \rightarrow \diamond_{n_{1}} a \vee \diamond_{n_{2}} b} \\
a \vee b \rightarrow c \\
(A 2) \frac{a \wedge b \rightarrow d}{\diamond_{n_{1}} a \wedge \diamond_{n_{2}} b \rightarrow \diamond_{n_{1}+n_{2}+1} c \vee \diamond_{0} d} \quad(R N) \frac{\neg a}{\neg \diamond_{0} a}
\end{gathered}
$$

$((G 1)$ and $(N)$ are easily derived from $(R G 1)$ and $(R N)$, respectively; $(G 2)$ follows by $(A 1)$ taking $n_{2}=0$; and $(G 3)$ may be derived using $(A 1)$ and $\left.(A 2)\right)$. All these rules are subsumed by the rule schema

$$
\text { (G) } \frac{\sum_{i=1}^{n} a_{i} \leq \sum_{j=1}^{m} b_{j}}{\bigwedge_{i=1}^{n} \diamond_{k_{i}} a_{i} \rightarrow \bigvee_{j=1}^{m} \diamond_{l_{j}} b_{j}},
$$

where $n, m \geq 0$, subject to the side condition $\sum_{i=1}^{n}\left(k_{i}+1\right) \geq 1+\sum_{j=1}^{m} l_{j}$ (which entails that $n$ and $m$ cannot both be 0 ). One-step soundness of $(G)$ follows from onestep soundness of the rule system for majority logic proved in the next example. By the preceding considerations, $(G)$ is weakly complete, and hence one-step complete by Proposition 5.3.

Resolution closedness of $(G)$ : Take two instances of $(G)$, one denoted like in the general form of the rule and one with all entities primed ( $a_{i}^{\prime}$ etc.), with the resolution taking place w.l.o.g. by matching $\diamond_{k_{1}^{\prime}} a_{1}^{\prime} \equiv \diamond_{l_{1}} b_{1}$. The conclusion of the arising resolvent is

$$
\bigwedge_{i=1}^{n} \diamond_{k_{i}} a_{i} \wedge \bigwedge_{i=2}^{m^{\prime}} \diamond_{k_{i}^{\prime}} a_{i}^{\prime} \rightarrow \bigvee_{i=2}^{m} \diamond_{l_{j}} b_{j} \vee \bigvee_{j=1}^{m^{\prime}} \diamond_{l_{j}^{\prime}} b_{j}^{\prime}
$$

Since $a_{1}^{\prime} \equiv b_{1}$, the premises $\sum_{i=1}^{n} a_{i} \leq \sum_{j=1}^{m} b_{j}$ and $\sum_{i=1}^{n^{\prime}} a_{i}^{\prime} \leq \sum_{j=1}^{m^{\prime}} b_{j}^{\prime}$ imply

$$
\sum_{i=1}^{n} a_{i}+\sum_{i=2}^{n^{\prime}} a_{i}^{\prime} \leq \sum_{j=2}^{m} b_{j}+\sum_{j=1}^{m^{\prime}} b_{j}^{\prime}
$$

and since $k_{1}=l_{1}^{\prime}$, the side conditions $\sum_{i=1}^{n}\left(k_{i}+1\right) \geq 1+\sum_{j=1}^{m} l_{j}$ and $\sum_{i=1}^{n^{\prime}}\left(k_{i}^{\prime}+1\right) \geq$ 
$1+\sum_{i=1}^{m^{\prime}} l_{j}^{\prime}$ imply

$$
\sum_{i=1}^{n}\left(k_{i}+1\right)+\sum_{i=2}^{n^{\prime}}\left(k_{i}^{\prime}+1\right) \geq 1+\sum_{j=2}^{m} l_{j}+\sum_{j=1}^{m^{\prime}} l_{j}^{\prime},
$$

so that we arrive again at an instance of $(G)$.

(7) Majority logic: in [Pacuit and Salame 2004], the extension of the axiomatization of graded modal logic with the axioms

$$
\begin{aligned}
& M a \wedge M b \rightarrow \diamond_{0}(a \wedge b) \\
& M a \wedge \square_{0}(a \rightarrow b) \rightarrow M b \\
& W a \wedge W b \wedge \diamond_{n}(\neg a \wedge \neg b) \rightarrow \diamond_{n}(a \wedge b) \\
& W a \wedge M b \wedge \diamond_{n}(\neg a \wedge \neg b) \rightarrow \diamond_{n+1}(a \wedge b)
\end{aligned}
$$

is proved to be weakly complete for majority logic including propositional symbols. These axioms are derivable from the set of rules

$$
\begin{array}{cc}
(R M 1) \frac{a \vee b}{W a \vee W b} & (R M 2) \frac{a \rightarrow b \vee c}{W a \rightarrow W b \vee \diamond_{0} c} \\
\neg(a \wedge c) & \neg a \wedge b \\
\neg(b \wedge c) & a \rightarrow c \vee d \\
(R M 3) \frac{a \wedge b \rightarrow d}{W a \wedge W b \wedge \diamond_{n} c \rightarrow \diamond_{n} d} & (R M 4) \frac{b \rightarrow c}{W a \wedge \diamond_{n} b \rightarrow W c \vee \diamond_{n+1} d}
\end{array}
$$

$((M 2),(M 3)$ and (M4) follow directly from (RM2), (RM3) and (RM4), respectively; $(R M 1)$ proves $M a \rightarrow W a$, whence $(M 1)$ is obtained from $(R M 2))$. These rules and rule $(G)$ for GML are subsumed by the rule schema

$$
\left(M_{u}\right) \frac{\sum_{i=1}^{n} a_{i}+\sum_{r=1}^{v} c_{r}+u \leq \sum_{j=1}^{m} b_{j}+\sum_{s=1}^{w} d_{s}}{\bigwedge_{i=1}^{n} \diamond_{k_{i}} a_{i} \wedge \bigwedge_{r=1}^{v} W c_{r} \rightarrow \bigvee_{j=1}^{m} \diamond_{l_{j}} b_{j} \vee \bigvee_{s=1}^{w} W d_{s}}(u \in \mathbb{Z})
$$

with side conditions $\sum_{i=1}^{n}\left(k_{i}+1\right)-\sum_{j=1}^{m} l_{j}-1+w-\max (u, 0) \geq 0$ and $v-w+2 u \geq 0$ (take $u=1$ for $(R M 1), u=0$ for $(R M 2),(R M 4)$, and $(G)$, and $u=-1$ for $(R M 3))$. Resolution closedness is checked analogously as for graded modal logic, covering the two cases of resolution at literals $\diamond_{n} a$ and $W a$, respectively; in both cases, an instance of $M_{u_{1}+u_{2}}$ can be taken as a resolvent of an instance of $M_{u_{1}}$ and an instance of $M_{u_{2}}$.

One-step soundness of $\left(M_{u}\right)$ : Let $\tau$ be a $\mathcal{P}(X)$-valuation such that $X, \tau \models$ $\sum_{i=1}^{n} a_{i}+\sum_{r=1}^{v} c_{r}+u \leq \sum_{j=1}^{m} b_{j}+\sum_{s=1}^{w} d_{s}$. Let $B \in \mathcal{B}(X)$. Using Lemma 3.17, we obtain by summation over $x \in X$

$$
\sum_{i=1}^{n} B\left(\sigma\left(a_{i}\right)\right)+\sum_{r=1}^{v} B\left(\sigma\left(c_{r}\right)\right)+u B(X) \leq \sum_{j=1}^{m} B\left(\sigma\left(b_{j}\right)\right)+\sum_{s=1}^{w} B\left(\sigma\left(d_{s}\right)\right) .
$$

Now put $p=\lceil B(X) / 2\rceil$ (with $\lceil x\rceil=\min \{z \in \mathbb{Z} \mid z \geq x\}$ ) so that $B$ satisfies $W a$ iff $B(\tau(a)) \geq p$. To establish that $B$ is in the interpretation of the conclusion of $M_{u}$, 
it suffices to prove

$$
\sum_{i=1}^{n}\left(k_{i}+1\right)+v p+u B(X) \geq \sum_{j=1}^{m} l_{j}+w(p-1)+1 .
$$

By the side conditions, this inequality is equivalent to

$$
-2 u p+u B(X)+\max (u, 0) \geq 0,
$$

which is easily established by distinguishing the cases $B(X)=2 p$ and $B(X)=$ $2 p-1$.

(8) Probabilistic modal logic: By reformulating the one-step complete set of axioms for probabilistic modal logic given by Cîrstea and Pattinson [2007] as one-step rules and subsequently applying resolution, one obtains the rules

$$
\left(P_{u}\right) \frac{\sum_{i=1}^{n} a_{i}+u \leq \sum_{j=1}^{m} b_{j}}{\bigwedge_{i=1}^{n} L_{p_{i}} a_{i} \rightarrow \bigvee_{j=1}^{m} L_{q_{j}} b_{j}},
$$

where $m, n \geq 0, m+n \geq 1$, and $u \in \mathbb{Z}$, subject to the side condition

$$
\begin{aligned}
& \sum_{i=1}^{n} p_{i}+u \geq \sum_{j=1}^{m} q_{j} \text { and } \\
& \sum_{i=1}^{n} p_{i}+u>0 \quad \text { if } m=0 .
\end{aligned}
$$

One-step completeness of $\left(P_{u}\right)$ : The rule schema is one-step complete, as it subsumes the following axiomatisation that has been shown to be one-step complete in loc.cit.:

$$
\begin{gathered}
\text { (0) } L_{0} a \quad(\top) \frac{a}{L_{p} a} \quad(>1) \frac{\neg a \vee \neg b}{\neg L_{p} a \vee \neg L_{q} b}(p+q>1) \\
\text { (1) } \frac{a \vee b}{L_{p} a \vee L_{q} b}(p+q=1) \\
\text { (1) } \frac{\sum_{i=1}^{r} c_{i}=\sum_{j=1}^{s} \bar{d}_{j}}{\bigwedge_{i=1}^{r} L_{u_{i}} c_{i} \wedge \bigwedge_{j=2}^{s} L_{\left(1-v_{j}\right)} d_{j} \rightarrow L_{v_{1}} d_{1}}
\end{gathered}
$$

where $\bar{d}_{1}=d_{1}$ and $\bar{d}_{j}=\neg d_{j}$ for $j \geq 2$, and rule $(\mathbb{1})$ is subject to the side condition

$$
\sum_{j=1}^{s} v_{j}=\sum_{i=1}^{r} u_{i}
$$

These rules are subsumed by the rule schema $\left(P_{u}\right)$, as follows. Rule $(0)$ : take $m=1, n=0, u=0, q_{1}=0$. Rule $(\top)$ : take $m=1, n=0, u=1$. Rule $>1$ : take $n=2, m=0, u=-1$. Rule (1): take $n=0, m=2, u=-1$. Rule ( $\mathbb{1}$ ): take $m=1, n=r+s-1, u=1-s$, and instantiate $b_{i}$ to $c_{i}$ for $i=1, \ldots, r, b_{i}$ to $d_{i-r+1}$ for $i=r+1, \ldots, r+s-1, a_{1}$ to $d_{1}, q_{i}$ to $u_{i}$ for $i=1, \ldots, r, q_{i}$ to $1-v_{i-r+1}$ for $i=r+1, \ldots, r+s-1$, and $p_{1}$ to $v_{1}$.

One-step soundness: Analogously to the previous example, using additionally that one always has $P(X)=1$.

Resolution closedness: Analogously as for graded modal logic; as a resolvent of an instance of $P_{u_{1}}$ and an instance of $P_{u_{2}}$, one can take an instance of $P_{u_{1}+u_{2}}$. 


\section{THE SHALLOW MODEL CONSTRUCTION}

We now present the announced generic shallow model construction, which is based on strictly one-step complete axiomatisations. The construction generalises results from [Vardi 1989] (where the use of axiomatisations is implicit in certain lemmas).

Definition 4.1. The set $M A(\phi)$ of (top level) modal atoms of a formula $\phi$ is defined recursively by $M A(\phi \wedge \psi)=M A(\phi) \cup M A(\psi), M A(\neg \phi)=M A(\phi)$, and $M A(L \rho)=\{L \rho\}$. (Note that $\phi \in \operatorname{Prop}(M A(\phi))$.) A pseudovaluation is a conjunctive clause $H$ over $\operatorname{Up}(\mathcal{F}(\Lambda)$ ), represented as a set of literals (i.e. pseudovaluations are identified modulo contraction and reordering of literals, which does not affect the set $M A(H)$ of modal atoms). A pseudovaluation is consistent if it is consistent as an $\mathcal{F}(\Lambda)$-formula. We say that $H$ is a pseudovaluation for $\phi$ if $M A(H) \subseteq M A(\phi)$ and $H \vdash_{P L} \phi$. If $\phi / \psi$ is a rule in $\mathcal{R}_{C}$ and $\sigma$ is a substitution such that $\psi \sigma \in \mathrm{Cl}(M A(H))$ and $H \vdash_{P L} \neg \psi \sigma$, then the negated instance $\neg \phi \sigma$ of the premise $\phi$ is a demand of $H$.

This generalises the notion of demand [Blackburn et al. 2001, Definition 6.43] to a coalgebraic setting. Note that by the dual of Lemma 3.2, all demands of a pseudovaluation $H$ are contained in $H$ when regarded as sets of literals, unless $H$ is propositionally inconstent (i.e. contains both $L \rho$ and $\neg L \rho$ for some modal atom $L \rho$ ).

LEMMA 4.2. Every consistent formula has a consistent pseudovaluation.

Proof. If $\phi$ is consistent, then one of the conjunctive clauses from its disjunctive normal form (DNF) is consistent and hence is a consistent pseudovaluation for $\phi$.

LEMma 4.3. Every demand of a consistent pseudovaluation is consistent.

Proof. By contraposition: Let $H$ be a pseudovaluation, and let $\phi / \psi$ be a rule in $\mathcal{R}_{C}$ such that $\psi \sigma \in \mathrm{Cl}(M A(H))$ and $H \vdash_{P L} \neg \psi \sigma$. If the demand $\neg \phi \sigma$ is inconsistent, then $\phi \sigma$ is provable; hence, $\psi \sigma$ is provable using $\phi / \psi$, and consequently $H$ is inconsistent.

Definition 4.4. A supporting Kripke frame of a $T$-coalgebra $(X, \xi)$ is a Kripke frame $(X, \mathcal{K})$ (consisting of a set $X$ and a transition relation $\mathcal{K} \subseteq X \times X)$ such that for each $x \in X$,

$$
\xi(x) \in T\{y \mid x \mathcal{K} y\} \subseteq T X .
$$

Lemma And Definition 4.5. If a coalgebra $C=(X, \xi)$ is equipped with a supporting Kripke frame $(X, \mathcal{K})$, then for every state $x \in X$, the set $X_{x}$ of states reachable from $x$ in $(X, \mathcal{K})$ is the carrier of a subcoalgebra $C_{x}=\left(X_{x}, \xi_{x}\right)$ of $C$, the submodel generated by $x$.

Note that by Proposition 2.5, $y \models_{C_{x}} \phi$ iff $y \models_{C} \phi$ for $y \in X_{x}$.

Definition 4.6. A shallow tableau is a Kripke frame $(X, \mathcal{K})$ with a distinguished root $H_{0} \in X$ such that $X$ is a set of pseudovaluations, every state is reachable from $H_{0}$, for all $H, G \in X$,

$$
H \mathcal{K} G \Longrightarrow G \text { is a pseudovaluation for a demand of } H,
$$

and for every demand $\phi$ of $H \in X$ there exists a pseudovaluation $G \in X$ for $\phi$ such that $H \mathcal{K} G$. Given a formula $\phi$, a shallow tableau for $\phi$ is a shallow tableau whose root is a pseudovaluation for $\phi$. 
A shallow tableau model is a $T$-coalgebra $C=(X, \xi)$ which has a supporting Kripke frame $(X, \mathcal{K})$ such that $(X, \mathcal{K})$ is a shallow tableau and the truth lemma

$$
H \vdash_{P L} \chi \Longrightarrow H \models_{C} \chi \quad \text { for all } \mathcal{F}(\Lambda) \text {-formulas } \chi
$$

holds for all $H \in X$ (hence in particular $H \models_{C} \chi$ if $H$ is a pseudovaluation for $\chi$ ).

A shallow tableau is almost a dag, except that in the presence of the rule $\perp / \perp$ (cf. Section 3) the pseudovaluation $T$ is a pseudovaluation for one of its own demands. Explicitly:

Proposition 4.7. A shallow tableau $(X, \mathcal{K})$ with root $H_{0}$ is, up to a possible loop at the state $T$, a dag of depth at most the depth of $H_{0}$, and the branching degree at $H \in X$ is exponentially bounded in $|H|$.

Proof. The first claim follows from the fact the the depth of all demands of a pseudovaluation $H$ is strictly less than the depth of $H$. To prove the bound on branching, note that pseudovaluations for demands of $H$ are conjunctive clauses over the set of subformulas of $H$.

Lemma 4.8. If a formula $\phi$ has a pseudovaluation $H_{0}$ such that all demands of $H_{0}$ are consistent, then there exists a shallow tableau for $\phi$.

(By Lemmas 4.2 and 4.3, the conditions of the above lemma hold in particular if $\phi$ is satisfiable.)

Proof. Let $Z$ consist of $H_{0}$ and all consistent pseudovaluations, and for $H, G \in$ $Z$ put $H \overline{\mathcal{K}} G$ iff $G$ is a pseudovaluation for a demand of $H$. Let $(X, \mathcal{K})$ be the subframe of $(Z, \overline{\mathcal{K}})$ generated by $H_{0}$ (i.e. $X$ is the set of states reachable from $H_{0}$ in $(Z, \overline{\mathcal{K}})$, and $\mathcal{K}=\overline{\mathcal{K}} \cap(X \times X))$. By the assumption on $H_{0}$ and Lemmas 4.2 and 4.3, $(X, \mathcal{K})$ is a shallow tableau for $\phi$.

THEOREM 4.9. If $\mathcal{R}$ is strictly one-step complete, then every shallow tableau is a supporting Kripke frame of a shallow tableau model.

Proof. Let $(X, \mathcal{K})$ be a shallow tableau; we have to construct a shallow tableau model $C=(X, \xi)$ for which $(X, \mathcal{K})$ is a supporting Kripke frame. To begin, note that to ensure the truth lemma, it suffices that $C$ is coherent in the sense that for $H \in X$ and $Y=\{G \mid H \mathcal{K} G\}$,

$$
H \vdash_{P L} L \rho \Longleftrightarrow \xi(H) \in \llbracket L \rrbracket_{Y}\left\{G \in Y \mid G \models_{C_{G}} \rho\right\} \text { for all } L \rho \in M A(H)
$$

(cf. Lemma and Definition 4.5) : note that $\left\{G \in Y \mid G \models_{C_{G}} \rho\right\}=\llbracket \rho \rrbracket_{C} \cap Y$, so that by naturality of predicate liftings, coherence implies that

$$
H \vdash_{P L} L \rho \Longleftrightarrow H \models_{C} L \rho \text { for all } L \rho \in M A(H) .
$$

The extension to propositional consequences of $H$ is then straightforward (noting that for $L \rho \in M A(H)$, either $H \vdash_{P L} L \rho$ or $\left.H \vdash_{P L} \neg L \rho\right)$.

We construct a coherent coalgebra structure $\xi$ by induction over the depth of pseudovaluations. Thus, let $H \in X$, put $Y=\{G \mid H \mathcal{K} G\}$, and assume that $\xi$ is already constructed for all pseudovaluations of smaller depth in $X$, in particular for all states $G$ reachable from $H$ in $(X, \mathcal{K})$. Thus, the submodel $C_{G}$ generated by such 
a state $G$ is already defined, and coherence at $G$ is unaffected by the construction of $\xi(H)$.

We have to prove that there exists $\xi(H) \in T Y \subseteq T X$ satisfying the coherence condition. Assume the contrary. Let $V$ be the set of propositional variables $b_{\rho}$, where $L \rho \in M A(H)$ for some $L$. Let $\theta \in \mathrm{Cl}(\mathrm{Up}(V))$ consist of the literals $\neg L b_{\rho}$ for $L \rho \in H$ and $L b_{\rho}$ for $\neg L \rho \in H$. By assumption, $T Y, \tau^{Y} \models \theta$, where $\tau^{Y}$ is the $\mathcal{P}(Y)$-valuation taking $b_{\rho}$ to $\left\{G \in Y \mid G \models_{C_{G}} \rho\right\}$. By strict one-step completeness, it follows that $\psi \eta \vdash_{P L} \theta$ for a rule $\phi / \psi$ in $\mathcal{R}_{C}$ and a $V$-substitution $\eta$ such that $Y, \tau^{Y} \models \phi \eta$. By construction of $\theta, H \vdash_{P L} \neg \theta \sigma$ and hence $H \vdash_{P L} \neg \psi \eta \sigma$. Thus, $\neg \phi \eta \sigma$ is a demand for $H$, and hence there exists in $Y$ a pseudovaluation $G$ for $\neg \phi \eta \sigma$. By the truth lemma for $G, G \models_{C_{G}} \neg \phi \eta \sigma$, in contradiction to $Y, \tau^{Y} \models \phi \eta$.

COROLlary 4.10. If $\mathcal{R}$ is strictly one-step complete, then the following are equivalent for an $\mathcal{F}(\Lambda)$-formula $\phi$.

(1) $\phi$ is satisfiable.

(2) $\phi$ is consistent.

(3) $\phi$ has a pseudovaluation $H$ such that all demands of $H$ are consistent.

(4) $\phi$ has a pseudovaluation $H$ such that all demands of $H$ are satisfiable.

(5) There exists a shallow tableau for $\phi$.

(6) $\phi$ is satisfiable at the root of a shallow tableau model.

Proof. (1) $\Longrightarrow$ (2): By soundness.

(2) $\Longrightarrow$ (3): By Lemmas 4.2 and 4.3

(3) $\Longrightarrow(5)$ : By Lemma 4.8.

$(5) \Longrightarrow(6)$ : By Theorem 4.9.

$(6) \Longrightarrow(1)$ : Trivial.

(3) $\Longleftrightarrow(4)$ : By the equivalence $(1) \Longleftrightarrow(2)$ already established.

The above implies in particular that the proof system is weakly complete, i.e. proves all valid formulas; this reproves a result of [Pattinson 2003]. By Remark 3.8, we obtain moreover that coalgebraic modal logic has the shallow model property:

Corollary 4.11 Shallow model Property. Every satisfiable $\mathcal{F}(\Lambda)$ formula $\phi$ is satisfiable in a shallow model, i.e. in a T-coalgebra that has a supporting Kripke frame $(X, \mathcal{K})$ which has final state $x_{\top}$, i.e. $x_{\top} \mathcal{K} x$ implies $x=x_{\top}$, and which, up to a possible loop at $x_{\top}$, is a dag of depth at most the depth of $\phi$ and of size at most $3^{n}$, where $n$ is the number of subformulas of $\phi$.

Proof. All that remains to be checked is the bound on the size: every state in a shallow tableau is a set representing a conjunctive clause over subformulas of $\phi$, in which a given subformula may occur as a positive literal, as a negative literal, or not at all.

\section{SHALLOW PROOFS}

The satisfiability criterion of Corollary 4.10 can be rephrased in terms of a shallow proof property. This property can be proved semantically by dualising Corollary 4.10, as done in the proof of Corollary 5.1 below. Alternatively, the shallow proof property can be established purely syntactically, without any reference to 
models; we present such an argument in the proof of Theorem 5.2 below. The shallow model construction presented in the previous section is however of independent interest.

Corollary 5.1 Shallow Proof Property. Let $\mathcal{R}$ be strictly one-step complete. Then an $\mathcal{F}(\Lambda)$-formula $\phi$ is provable iff for each clause $\rho$ in the conjunctive normal form (CNF) of $\phi$, there exists a rule $\chi / \psi \in \mathcal{R}_{C}$ and a substitution $\sigma$ such that $\psi \sigma \vdash_{P L} \rho$ and $\chi \sigma$ is provable.

Proof. The 'if' direction is trivial; we prove 'only if'. Dualizing the implication $(3) \Longrightarrow(2)$ in Corollary 4.10 yields

if $\phi$ is provable then each pseudovaluation $H$ for $\neg \phi$ has a demand $\chi$ such that $\neg \chi$ is provable.

Now let $\rho$ be a clause in the CNF of $\phi$. Then $\neg \rho$ is a conjunctive clause in the DNF of $\neg \phi$, in particular a pseudovaluation for $\neg \phi$. By the above condition, there exists a rule $\chi / \psi \in \mathcal{R}_{C}$ and a substitution $\sigma$ such that $\neg \rho \vdash_{P L} \neg \psi \sigma$, hence $\psi \sigma \vdash_{P L} \rho$, and $\chi \sigma$ is provable.

In a purely syntactic formulation of the shallow proof property, we have to replace strict completeness by closedness under resolution. The statement thus takes the following form.

Theorem 5.2 Shallow Proof Property. Let $\mathcal{R}$ be resolution closed. Then an $\mathcal{F}(\Lambda)$-formula $\phi$ is provable under $\mathcal{R}$ iff for each clause $\rho$ in the CNF of $\phi$, there exists a rule $\chi / \psi \in \mathcal{R}_{C}$ and a substitution $\sigma$ such that $\psi \sigma \vdash_{P L} \rho$ and $\chi \sigma$ is provable.

(This reproves Corollary 5.1, as strict one-step completeness implies resolution closedness by Theorem 3.16.)

ProOF. Again, 'if' is trivial, and we prove 'only if'. Let $\phi$ be provable, and let $\rho$ be a clause in the CNF of $\phi$. Then $\rho$ is provable. By definition of the proof system, $\rho$ is propositionally entailed by the set of clauses

$$
\Phi=\left\{\psi \sigma \mid \chi / \psi \in \mathcal{R}_{C}, \chi \sigma \text { provable }\right\}
$$

One shows analogously as in the 'if' direction of the proof of Theorem 3.16 that $\Psi$ is resolution closed. By Lemma 3.14, there exists $\psi \sigma$ in $\Phi$ such that $\psi \sigma \vdash_{P L} \rho$.

We hope that both proofs of the shallow proof property provide a handle for generalizations to logics outside rank 1.

One application of the shallow proof property is

Proposition 5.3. Let $\Lambda$ contain an infinite set $U$ of propositional symbols, modelled as in Remark 2.8 over a functor $T_{U}$ of the form $T_{U} X=T X \times \mathcal{P}(U)$. Then the proof system induced by $\mathcal{R}$ is weakly complete iff $\mathcal{R}$ is one-step complete.

Proof. W.l.o.g. $\mathcal{R}$ is resolution closed (one can close under resolution, thereby affecting neither completeness nor one-step completeness). The 'if' direction is known (cf. Remark 3.8). To prove the 'only if' direction, let $\psi \in \mathrm{Cl}(\mathrm{Up}(V))$, let $X$ be a set, and let $\tau$ be a $\mathcal{P}(X)$-valuation such that $T_{U} X, \tau \models \psi$. Since $U$ is infinite and $V$ may be assumed to be finite, we can assume w.l.o.g. that $V \subseteq U$. Let $\phi$ denote 
the propositional theory of $\tau$, i.e. the conjunction of all contracted clauses $\chi$ over $V$ such that $X, \tau \models \chi$. Then one checks as in the proof of Theorem 17 in [Schröder 2007] that the rule $\phi / \psi$ is one-step sound. By Lemma 16 in [Schröder 2007], there exists a $\operatorname{Prop}(V)$-substitution $\sigma$ such that $\phi \sigma$ and $\phi \rightarrow(a \leftrightarrow \sigma(a))$ (for each $a \in V$ ) are propositional tautologies. Since $V \subseteq U$, we can regard $\phi \sigma$ as an $\mathcal{F}(\Lambda)$-formula. As such, $\phi \sigma$ is valid. By soundness of $\phi / \psi$, it follows that $\psi \sigma$, again regarded as an $\mathcal{F}(\Lambda)$-formula, is valid, hence provable by weak completeness. By the shallow proof property (Theorem 5.2), there exist a rule $\chi / \rho$ over $W$ and a $\operatorname{Prop}(V)$-substitution $\theta$ such that $\chi \theta$ is provable and $\rho \theta \vdash_{P L} \psi \sigma$. By Lemma 3.2 and Assumption 3.10, it follows that there exists a $V$-substitution $\kappa$ such that $\sigma(\kappa(b))=\theta(b)$ for all $b \in W$ and $\rho \kappa \vdash_{P L} \psi$.

It remains to prove that $X, \tau \models \chi \kappa$. From $X, \tau \models \phi$ and the construction of $\sigma$, we obtain $X, \tau \models a \leftrightarrow \sigma(a)$ for all $a \in V$ and hence $X, \tau \models \kappa(b) \leftrightarrow \theta(b)$ for all $b \in W$, so that the goal follows from $X, \tau \models \chi \theta$.

Remark 5.4. In the above result, the assumption that $\Lambda$ contains enough propositional symbols is essential. E.g. in cases like coalition logic or probabilistic modal logic where the logic collapses into triviality without propositional symbols, the empty set of rules is complete, but not one-step complete.

The proof-theoretic content of Theorem 5.2 goes beyond the mere fact that proofs are shallow. The theorem asserts that if the rule system is resolution closed, then propositional reasoning can always be limited to decomposing a formula into the clauses of its CNF and propositional entailment (i.e. by Lemma 3.2 essentially containment) between clauses. Moreover, shallow proofs witness a weak subformula property: every provable formula has a proof that mentions only propositional combinations of subformulas. Formally:

TheOrem 5.5 Weak Subformula Property. Suppose that $\mathcal{R}$ is resolution closed and $\phi$ is derivable under $\mathcal{R}$. Then there exists a proof of $\phi$ that mentions only propositional combinations of subformulas of $\phi$.

Proof. Assume that $\phi$ is derivable under $\mathcal{R}$ and $\rho$ is a clause of the CNF of $\phi$; w.l.o.g. $\rho$ is not a tautology. By Theorem 5.2 we find a rule $\chi / \psi \in \mathcal{R}$ and a substitution $\sigma$ such that $\psi \sigma \vdash_{P L} \rho$ and $\chi \sigma$ is provable under $\mathcal{R}$; by Lemma 3.2, $\rho$ contains $\psi \sigma$, hence we can assume w.l.o.g. that $\sigma$ maps propositional variables to subformulas of $\rho$. As $\chi$ is a purely propositional formula, the substituted premise $\chi \sigma$ is a propositional combinations of subformulas of $\rho$, hence also of $\phi$. The claim now follows inductively.

As a consequence, it is immediate that $\mathcal{F}(\Lambda)$ is a conservative extension of any sublanguage $\mathcal{F}\left(\Lambda_{0}\right)$ induced by a sub-signature $\Lambda_{0} \subseteq \Lambda$ :

Corollary 5.6 Conservativity. Suppose $\mathcal{R}$ is resolution closed, $\Lambda_{0} \subseteq \Lambda$ is a sub-signature and $\mathcal{R}_{0}$ consists of those $\phi / \psi \in \mathcal{R}$ that mention only modal operators in $\Lambda_{0}$. Then a formula $\phi \in \mathcal{F}\left(\Lambda_{0}\right)$ is $\mathcal{R}$-derivable iff it is $\mathcal{R}_{0}$-derivable.

In particular, if $\mathcal{R}$ is weakly complete for $\mathcal{F}(\Lambda)$, then $\mathcal{R}_{0}$ is weakly complete for $\mathcal{F}\left(\Lambda_{0}\right)$.

Example 5.7. From completeness of the rules $\left(M_{u}\right)$ for majority logic (Exam- 
ple 3.18.7), we obtain that the rules

$$
\left(W_{u}\right) \frac{\sum_{r=1}^{v} c_{r}+u \leq \sum_{s=1}^{w} d_{s}}{\bigwedge_{r=1}^{v} W c_{r} \rightarrow \bigvee_{s=1}^{w} W d_{s}}(u \in \mathbb{Z})
$$

with side conditions $w-1-\max (u, 0) \geq 0$ and $v-w+2 u \geq 0$ form a complete axiomatisation of the majority operator $W$ alone. (Pauly [2005] considers a similar language, but without nesting of modal operators in formulas.)

\section{A GENERIC PSPACE ALGORITHM}

We will now exploit the shallow model result (Corollary 4.10) to design a decision procedure for satisfiability in the spirit of [Vardi 1989]. This requires one more preparatory step: since resolution closed rule sets are in general infinite, we must ensure that we never need to instantiate a rule in such a way that the conclusion contains the same literal twice; otherwise, determining the demands of a given pseudovaluation (Definition 4.1) might require checking infinitely many rules. This is formally captured as follows.

Definition 6.1. An instance $\phi \sigma / \psi \sigma$ of a rule $\phi / \psi$ is contracted if the clause $\psi \sigma$ is contracted (Definition 3.1). In this case, if $H$ is a pseudovaluation (Definition 4.1) such that $\psi \sigma \in \mathrm{Cl}(M A(H))$ and $H \vdash_{P L} \neg \psi \sigma$, the demand $\neg \phi \sigma$ of $H$ is called an essential demand. We say that a set $\mathcal{R}$ of rules is closed under contraction if for every $V$-instance $\phi \sigma / \psi \sigma$ of a rule $\phi / \psi$ over $V$ in $\mathcal{R}$, there exists a contracted $V$ instance $\phi^{\prime} \sigma^{\prime} / \psi^{\prime} \sigma^{\prime}$ of a rule $\phi^{\prime} / \psi^{\prime} \in \mathcal{R}$ such that $\psi^{\prime} \sigma^{\prime}$ propositionally entails $\psi \sigma$ and $\phi \sigma$ propositionally entails $\phi^{\prime} \sigma^{\prime}$.

I.e. a rule set is closed under contraction if every instance of a rule that duplicates literals in the conclusion can be replaced by a contracted instance of a different rule. Not all the rule sets discussed in Example 3.18 satisfy this property, but they can easily be closed under contraction: just add a rule $\phi^{\prime} / \psi^{\prime}$ for every rule $\phi / \psi$ over $V$ in $\mathcal{R}$ and every $V$-substitution $\sigma$, where $\phi^{\prime}$ is some suitably chosen propositional equivalent of $\phi \sigma$ and $\psi^{\prime}$ is obtained from $\psi \sigma$ by removing duplicate literals. It is clear that the new rules remain one-step sound. Note that extending the rule set trivially preserves strict one-step completeness, so that there is no need to close the extended rule set under resolution again.

For convenience, we introduce further notation for propositional formulas: if $r \in \mathbb{Z}-\{0\}$ and $\phi$ is a formula, then we put

$$
\operatorname{sgn}(r) \phi= \begin{cases}\phi & r>0 \\ \neg \phi & r<0 .\end{cases}
$$

Example 6.2. (1) The strictly one-step complete rule sets of Examples 3.18.15 ( $E, M, K, K D$, and coalition logic) are easily seen to be closed under contraction, essentially because in all relevant rule schemas, the premise is a clause of the same general format as the conclusion.

(2) Graded modal logic: The rule schema $(G)$ of Example 3.18.6 fails to be closed under contraction, as duplicating literals in the conclusion substantially affects both the premise and the side condition. We can close $(G)$ under contraction as described 
above; this results in the rule schema

$$
\left(G^{\prime}\right) \frac{\sum_{i=1}^{n} r_{i} a_{i} \geq 0}{\bigvee_{i=1}^{n} \operatorname{sgn}\left(r_{i}\right) \diamond_{k_{i}} a_{i}}
$$

where $n \geq 1$ and $r_{1}, \ldots, r_{n} \in \mathbb{Z}-\{0\}$, subject to the side condition $\sum_{r_{i}<0} r_{i}\left(k_{i}+\right.$ 1) $\geq 1+\sum_{r_{i}>0} r_{i} k_{i}$.

(3) Majority logic: Similarly, closing the rule schema $\left(M_{m}\right)$ for majority logic under contraction yields the rule schema

$$
\left(M_{m}^{\prime}\right) \frac{m \leq \sum_{i=1}^{n} r_{i} a_{i}+\sum_{j=1}^{v} s_{j} b_{j}}{\bigvee \operatorname{sgn}\left(r_{i}\right) \diamond_{k_{i}} a_{i} \vee \bigvee \operatorname{sgn}\left(s_{j}\right) W b_{j}}\left(r_{i}, s_{j} \in \mathbb{Z}-\{0\}, m \in \mathbb{Z}\right)
$$

with side conditions $\sum_{r_{i}<0} r_{i}\left(k_{i}+1\right)-\left(\sum_{r_{i}>0} r_{i} k_{i}\right)-1+\sum_{s_{j}>0} s_{j}-\max (m, 0) \geq 0$ and $2 m-\sum s_{j} \geq 0$.

(4) Probabilistic modal logic: The rule schema $\left(P_{k}\right)$ of Example 3.18.8 fails to be closed under contraction. Closure under contraction as described above leads to the rule schema

$$
\left(P_{k}^{\prime}\right) \frac{\sum_{i=1}^{n} r_{i} a_{i} \geq k}{\bigvee_{1 \leq i \leq n} \operatorname{sgn}\left(r_{i}\right) L_{p_{i}} a_{i}}
$$

where $n \geq 1$ and $r_{1}, \ldots, r_{n} \in \mathbb{Z}-\{0\}$, subject to the side condition

$$
\begin{aligned}
\sum_{i=1}^{n} r_{i} p_{i} & \leq k, \text { and } \\
\text { if } \forall i . r_{i}<0 \text { then } \sum_{i=1}^{n} r_{i} p_{i} & <k .
\end{aligned}
$$

The crucial property of contraction closed rule sets is

LEMma 6.3. If $\mathcal{R}$ is closed under contraction, then all the demands of a pseudovaluation are satisfiable iff all its essential demands are satisfiable.

Proof. The 'only if' direction is trivial. We prove 'if': Let $\mathcal{R}$ be closed under contraction. Then also $\mathcal{R}_{C}$ is closed under contraction, since instances of the congruence rule never contain duplicate literals. Thus, every demand of a pseudovaluation $H$ is propositionally entailed by an essential demand.

Thus we can extend Corollary 4.10 as follows.

COROLLARY 6.4. If $\mathcal{R}$ is strictly one-step complete and closed under contraction, then an $\mathcal{F}(\Lambda)$-formula $\phi$ is satisfiable iff $\phi$ has a pseudovaluation $H$ such that all essential demands of $H$ are satisfiable.

In the algorithm suggested by Corollary 6.4, we will encode demands, which are themselves too large to be passed around directly, by the rules that induce them. Here, we need to represent rules by suitable codes, i.e. strings over some alphabet, since a naive direct representation of rules would in particular have to deal with rule premises of potentially exponential size.

Definition 6.5. We say that a rule $R \in \mathcal{R}$ matches a clause $\rho \equiv \bigvee_{i=1}^{n} \epsilon_{i} L_{i} \phi_{i}$ if the conclusion of $R$ is of the form $\bigvee_{i=1}^{n} \epsilon_{i} L_{i} a_{i}$. In this case, let $\sigma(R, \rho)$ denote the arising substitution $\left[\phi_{i} / a_{i}\right]_{i=1, \ldots, n}$. Two rules matching the same clause are equivalent if their premises are propositionally equivalent; equivalence classes $[R]$ are called $\mathcal{R}$-matchings. The code of $R$ is also a code for $[R]$. 
We fix some size measures for the representation of formulas and rules:

Definition 6.6. The size size $(a)$ of an integer $a$ is $\left\lceil\log _{2}(|a|+1)\right\rceil$, where $\lceil r\rceil=$ $\min \{z \in \mathbb{Z} \mid z \geq r\}$ as usual. The size size $(p)$ of a rational number $p=a / b$, with $a, b$ relatively prime, is $1+\operatorname{size}(a)+\operatorname{size}(b)$. The size $|\phi|$ of a formula $\phi$ over $V$ is defined by counting 1 for each propositional variable, boolean operator, or modal operator, and additionally the size of each index of a modal operator. (In the examples, indices are either numbers, with sizes as above, or subsets of $\{1, \ldots, n\}$, assumed to be of size $n$.)

Assumption 6.7. We assume a reasonable encoding of modal formulas in which boolean operators take up constant space and modal operators take up space according to a given coding of $\Lambda$; we assume that this coding is in $N P$ (i.e. it is decidable in $N P$ whether a given code is a valid code for a modal operator in $\Lambda$ ). Graded or probabilistic modal operators are assumed to be coded in binary, with sizes according to Definition 6.6.

Example 6.8. For the rules of Examples 3.18 and 6.2, we just take the parameters of a rule as its code in the obvious way. E.g. the code of an instance of $\left(P_{k}^{\prime}\right)$ as displayed in Example 6.2.4 consists of $n, k$, the $r_{i}$, and the $p_{i}$. The size of the code is determined by the sizes of these numbers plus separating letters, say, $\sum\left(1+\operatorname{size}\left(a_{i}\right)\right)+\sum\left(1+\operatorname{size}\left(p_{i}\right)\right)+\operatorname{size}(n)+\operatorname{size}(k)+1$. Note that not all such codes represent instances of $\left(P_{k}^{\prime}\right)$.

The following decision procedure on an alternating Turing machine generalises the PSPACE algorithms in [Vardi 1989], given a strictly one-step complete and contraction closed rule set $\mathcal{R}$.

Algorithm 6.9. (Decide satisfiability of $\phi \in \mathcal{F}(\Lambda)$ )

(1) (Existential) Guess a propositionally consistent pseudovaluation $H$ for $\phi$.

(2) (Universal) Choose a contracted clause $\perp \neq \rho$ over $M A(H)$ such that $H \vdash_{P L} \neg \rho$.

(3) (Universal) Choose an $\mathcal{R}_{C}$-matching $[R]$ of $\rho$.

(4) (Existential) Guess a clause $\gamma$ from the CNF of the premise of $R$.

(5) Recursively check that $\neg \gamma \sigma(R, \rho)$ is satisfiable.

The algorithm succeeds if all possible choices at steps marked universal lead to successful termination, and for all steps marked existential, there exists a choice leading to successful termination. Concerning Step 1, note that the only way for a pseudovaluation to be propositionally inconsistent is to contain both $L \rho$ and $\neg L \rho$ for some modal atom $L \rho$.

We emphasise that in Step 3, it suffices to guess one code for each matching.

Proposition 6.10. Algorithm 6.9 succeeds iff the input formula $\phi$ is satisfiable.

Proof. Induction over the depth $n$ of $\phi$. If $n=0$, then the propositional formula $\phi$ will evaluate to either $T$ or $\perp$, as it does not contain any propositional variables; moreover, the only candidate for a pseudovaluation for $\phi$ is the empty conjunctive clause $T$. Thus, the algorithm terminates unsuccessfully in the existential step (1) iff $\phi$ evaluates to $\perp$, since $T$ is a pseudovaluation for $\phi$ iff $\phi$ evaluates 
to $T$. Otherwise, the algorithm terminates successfully in the universal step (2), since the only clause $\rho$ over $M A(\top)=\emptyset$ such that $\top \vdash_{P L} \neg \rho$ is $\perp$. For $n>0$, correctness of the algorithm follows from Corollary 6.4 and the inductive hypothesis: the essential demands of $\phi$ are the negated premises $\neg \phi \sigma(\phi / \psi, \rho)$ for $\mathcal{R}_{C}$-matchings $[\phi / \psi]$ of contracted clauses $\rho$ as in the algorithm, and such a demand is satisfiable iff the negation of one of the clauses in the $\mathrm{CNF}$ of $\phi \sigma(\phi / \psi, \rho)$ is satisfiable.

Remark 6.11. In Step 1 of Algorithm 6.9, it suffices to consider the conjunctive clauses in some DNF of $\phi$ rather than all pseudovaluations. A canonical, if not necessarily the most effective choice for such a DNF is to take all pseudovaluations $H$ for $\phi$ such that $M A(H)=M A(\phi)$ (rather than only $M A(H) \subseteq M A(\phi)$ ); in a concrete implementation, a heuristic procedure for determining some DNF effectively may be preferable.

Note that due to the non-deterministic nature of the algorithm, the above proposition does not imply decidability of $\mathcal{F}(\Lambda)$. This follows only if the algorithm respects suitable resource bounds. We are interested in cases where the algorithm runs in polynomial time. The crucial requirement for this is that Steps 3 and 4 can be performed in polynomial time, i.e. by suitable nondeterministic polynomialtime multivalued functions (NPMV) [Book et al. 1984]. We recall that a function $f: \Sigma^{*} \rightarrow \mathcal{P}\left(\Delta^{*}\right)$, where $\Sigma$ and $\Delta$ are alphabets, is NPMV iff

(NPMV1) there exists a polynomial $p$ such that $|y| \leq p(|x|)$ for all $y \in f(x)$, where $|\cdot|$ denotes size, and

(NPMV2) the graph $\{(x, y) \mid y \in f(x)\}$ of $f$ is in $N P$.

This motivates the following conditions:

Definition 6.12. A set $\mathcal{R}$ of rules is called PSPACE-tractable if there exists a polynomial $p$ such that all $\mathcal{R}$-matchings of a contracted clause $\rho$ over $\mathcal{F}(\Lambda)$ have some code of size at most $p(|\rho|)$ (recall that matchings are equivalence classes of rules and thus may have several codes), and it can be decided in $N P$

(1) whether a given code is the code of some rule in $\mathcal{R}$;

(2) whether a rule matches a given contracted clause; and

(3) whether a clause belongs to the CNF of the premise of a given rule.

Theorem 6.13 Space Complexity. Let $\mathcal{R}$ be strictly one-step complete, closed under contraction, and PSPACE-tractable. Then the satisfiability problem for $\mathcal{F}(\Lambda)$ is in PSPACE.

Proof. Since $\mathcal{R}$ is $P S P A C E$-tractable, so is $\mathcal{R}_{C}$, assuming reasonable codes for the congruence rules (e.g. consisting of the representation of the relevant modal operator; cf. Assumption 6.7). Thus, the functions mapping a clause $\rho$ to the set of its $\mathcal{R}_{C}$-matchings and a rule to the set of clauses occurring in the CNF of its premise, respectively, are NPMV: in the former case, the polynomial bound required by condition (NPMV1) is ensured by the definition of PSPACE-tractability, as we only need to produce one code for each matching, and in the latter case, the polynomial bound holds universally, as clauses are of polynomial size. Condition (NPMV2) is ensured explicitly by Definition 6.12 and Assumption 6.7 (which implies that 
the set of formulas is in NP). Therefore, Steps 3 and 4 in Algorithm 6.9 can be performed in polynomial time. Steps 1 and 2 have polynomial runtime without specific assumptions, as a pseudovaluation $H$ for $\phi$ is represented as a set of literals and must by definition satisfy $M A(H) \subseteq M A(\phi)$, and the contracted clause $\rho$ chosen in Step 2 is constructed as a non-repetitive list of literals whose negations belong to $H$. Since the depth of recursion is bounded by the depth of $\phi$, it follows that the algorithm runs in APTIME = PSPACE [Chandra et al. 1981].

Remark 6.14. A more careful analysis of Algorithm 6.9 reveals that it suffices for the decision problems in Definition 6.12 to be in $P H$, the polynomial time hierarchy. In our examples, however, the complexity is in fact $P$ rather than $N P$. We expect that this situation is typical, with the crucial condition for PSPACE-tractability being the polynomial bound on $\mathcal{R}$-matchings. We are not aware of any natural examples of intractable rule sets (contrived examples are easy to construct, e.g. by imposing computationally hard side conditions).

Remark 6.15. Algorithm 6.9 can be dualised to yield a proof-search procedure that determines whether $\phi \in \mathcal{L}(\Lambda)$ is $\mathcal{R}$-derivable, thus implementing the shallow proof property (Corollary 5.1/Theorem 5.2). Note that the dualisation entails that the roles of existential and universal steps are interchanged.

In the treatment of graded and propositional modal logic, the polynomial bound on rule codes follows rather directly from size estimates in integer linear programming, as follows. Following usual practice, we take the size $|W|$ of a rational inequality $W \equiv\left(\sum_{i=1}^{n} u_{i} x_{i}\right.$ op $\left.u_{0}\right)$, op $\in\{<, \leq,>, \geq\}$ and $u_{i} \in \mathbb{Q}$, to be $1+n+\sum_{i=0}^{n} \operatorname{size}\left(u_{i}\right)$. We recall that for $n \in \mathbb{Z}, \operatorname{sgn}(n)=-1$ if $n<0, \operatorname{sgn}(n)=1$ if $n>0$, and $\operatorname{sgn}(n)=0$ if $n=0$.

Lemma 6.16. For every rational linear inequality $W$ and every solution $r_{0}, \ldots, r_{n} \in \mathbb{Z}$ of $W$, there exists a solution $s_{0}, \ldots, s_{n} \in \mathbb{Z}$ of $W$ such that $\operatorname{sgn}\left(s_{i}\right)=$ $\operatorname{sgn}\left(r_{i}\right)$ for all $i$, the propositional formulas $\sum_{i=1}^{n} s_{i} a_{i} \geq s_{0}$ and $\sum_{i=1}^{n} r_{i} a_{i} \geq r_{0}$ (cf. Section 3) are equivalent, and size $\left(s_{i}\right) \leq 18|W|^{4}$ for all $i$.

Proof. Let $V=\left\{a_{1}, \ldots, a_{n}\right\}$, and let $x_{0}, \ldots, x_{n}$ be the variables in $W$. We note that a propositional formula $\sum_{i=1}^{n} s_{i} a_{i} \geq s_{0}$ is equivalent to $\phi \equiv \sum_{i=1}^{n} r_{i} a_{i} \geq$ $a_{0}$ iff for all valuations $\nu: V \rightarrow\{0,1\}$, one has $\sum_{i=1}^{n} s_{i} \nu\left(a_{i}\right) \geq s_{0}$ if and only if $\sum_{i=1}^{n} r_{i} \nu\left(a_{i}\right) \geq r_{0}$, read as integer linear inequalities. Thus, let $I$ denote the system of inequalities consisting of $W$ and additional inqualities $F_{i}$ and $E_{\nu}$, where $i=1, \ldots, n, \nu$ ranges over valuations $V \rightarrow\{0,1\}$,

$$
F_{i}= \begin{cases}x_{i} \geq 1 & \text { if } r_{i} \geq 1 \\ x_{i}=0 & \text { if } r_{i}=0 \\ x_{i} \leq-1 & \text { if } r_{i} \leq-1\end{cases}
$$

(where the middle case actually corresponds to two inequalities), and

$$
E_{\nu}= \begin{cases}\sum_{i=1}^{n} x_{i} \nu\left(a_{i}\right) \geq x_{0} & \text { if } \sum_{i=1}^{n} r_{i} \nu\left(a_{i}\right) \geq r_{0} \\ \sum_{i=1}^{n} x_{i} \nu\left(a_{i}\right)<x_{0} & \text { if } \sum_{i=1}^{n} r_{i} \nu\left(a_{i}\right)<r_{0} .\end{cases}
$$

Then the claim translates into the statement that $I$ has a solution of polynomially bounded size in $|W|$. 
It follows from [Schrijver 1986, Corollary 17.1b] that $I$ has a solution whose size is bounded by $6 c(n+1)^{3}$, where $c$ is the facet complexity of the system, i.e. the size of the largest inequality in $I$. As the cofficients of the inequalities $E_{\nu}$ and $F_{i}$ are of size at most 1 , we have $c \leq|W|+2(n+1)$. Since moreover $|W| \geq n+1, I$ thus has a solution of size at most $18|W|^{4}$.

We now illustrate how Theorem 6.13 allows us to establish PSPACE bounds for many modal logics in a uniform way.

Example 6.17. Conditions (1) and (2) of Definition 6.12 are immediate for all the rule sets of Example 3.18 - the decision problems in question involve no more than checking computationally harmless side conditions in the case of Condition (1) (disjointness and containment of finite sets, linear inequalities), and comparing clauses of polynomial (in fact, linear) size in the case of Condition (2). Moreover, Condition (3) is immediate in those cases where the premises of rules are just single clauses. This leaves only GML and PML; but the expansion of $\sum_{i \in I} r_{i} a_{i} \geq k$ to a propositional formula is already in $\mathrm{CNF}$, and checking whether a given clause belongs to this CNF is clearly in $P$.

It remains to establish the polynomial bound on the matchings. For GML and PML, this is guaranteed precisely by Lemma 6.16. In all other cases, every contracted clause $\rho$ matches at most one rule, whose code has size linear in the size of $\rho$.

We thus have obtained PSPACE-tractability and hence decidability in PSPACE for all logics in Example 3.18. The logics $E$ and $M$ are of lesser interest here, being actually in $N P$ [Vardi 1989]. We briefly comment on the algorithms and bounds for the other cases.

(1) For the modal logics $K$ and $K D$ (Examples 3.18 .3 and 4), Algorithm 6.9 is essentially the witness algorithm [Ladner 1977; Vardi 1989; Blackburn et al. 2001]. Both logics are PSPACE-hard [Ladner 1977].

(2) For coalition logic (Example 3.18.5), we arrive, due to minor differences of the rule sets, at a slight variant of Pauly's PSPACE-algorithm [Pauly 2002].

(3) For graded modal logic, we obtain a new algorithm which confirms the known PSPACE upper bound [Tobies 2001]. One might claim that the new algorithm is not only nicely embedded into a unified framework, but also conceptually simpler than the constraint-based algorithm of [Tobies 2001] (which corrects a similar but incorrect algorithm previously given elsewhere, and refutes a previous EXPTIME hardness conjecture). Graded modal logic is PSPACE-hard, as it extends $K$.

(4) For probabilistic modal logic, we obtain a new algorithm which confirms the PSPACE upper bound that follows from the corresponding bound for the more expressive (modal) logic of probability, a proof of which is sketched in [Fagin and Halpern 1994]. The bound is tight, as PML contains the PSPACE-complete logic $K D$ as a fragment (embedded by mapping $\square$ to $L_{1}$ ). In comparison to the algorithm in loc. cit., our algorithm has additional proof theoretic content as discussed in Section 5. Under the correspondence outlined in Remark 6.15, it finds proofs which remain within PML rather than possibly diverting via a more expressive logic. 
(5) Our PSPACE upper bound for majority logic, which appeared for the first time in the conference presentation of [Schröder and Pattinson 2006], tied in a priority race with [Demri and Lugiez 2006], where a PSPACE upper bound was proved for the more expressive Presburger modal logic using a different type of algorithm. The same remarks concerning proof-theoretic content apply as for probabilistic modal logic.

\section{CONCLUSION}

Generalising results by Vardi [1989], we have shown that coalgebraic modal logic has the shallow model property, and we have presented a generic PSPACE algorithm for satisfiability based on depth-first exploration of shallow models. We have thus

—reproduced the witness algorithm for $K$ and $K D$ [Blackburn et al. 2001]

-obtained a slight variant of the known PSPACE algorithm for coalition logic [Pauly 2002]

- obtained a new PSPACE algorithm for graded modal logic, recovering the known PSPACE bound [Tobies 2001]

-obtained a new PSPACE algorithm for probabilistic modal logic [Larsen and Skou 1991; Heifetz and Mongin 2001], recovering a PSPACE upper bound which follows from results sketched in [Fagin and Halpern 1994].

- obtained, simultaneously with [Demri and Lugiez 2006], a new PSPACE upper bound for majority logic [Pacuit and Salame 2004].

In all these cases, the PSPACE upper bound is tight. Our algorithm may alternatively be viewed as traversing a shallow proof that witnesses a weak subformula property.

The crucial prerequisite for the generic algorithm is an axiomatisation by so-called one-step rules (going from rank 0 to rank 1) obeying two closedness conditions: closedness under resolution and under contraction, i.e. removal of duplicate literals. In the examples, it has not only turned out that it is feasible to keep this closure process under control, but also that the axiomatisations obtained have pleasingly compact presentations - typically, one ends up with a single rule schema.

It has been shown that every modal logic can be equipped with a canonical coalgebraic semantics, provided it is axiomatisable in rank 1 and satisfies the congruence rule [Schröder and Pattinson 2007b]. This means in particular that our shallow model construction applies to every such modal logic when equipped with the canonical semantics. Moreover, the PSPACE-algorithm presented here can be made modular w.r.t. heterogeneous combination of systems and modal logics using multi-sorted coalgebra [Schröder and Pattinson 2007a]. The extension of the theory beyond rank 1 is the subject of future research, as is the treatment of simple fixed point operators, possibly using automata theoretic methods [Vardi 1996; Venema 2006] or pseudomodels [Emerson and Halpern 1985]. A further point of interest is to investigate the connection between our notion of resolution closure and classical proof-theoretic issues such as cut elimination and interpolation. 


\section{ACKNOWLEDGMENTS}

The authors wish to thank Alexander Kurz for useful discussions and the Department of Computer Science at the University of Bremen for funding a visit of the second author.

\section{REFERENCES}

BArr, M. 1993. Terminal coalgebras in well-founded set theory. Theor. Comput. Sci. 114, 299315.

Bartels, F. 2003. Generalised coinduction. Math. Struct. Comput. Sci. 13, 321-348.

Bartels, F., Sokolova, A., And De Vink, E. P. 2004. A hierarchy of probabilistic system types. Theor. Comput. Sci. 327, 3-22.

Blackburn, P., De Rijke, M., And Venema, Y. 2001. Modal Logic. Cambridge University Press, Cambridge.

Book, R., Long, T., And Selman, A. 1984. Quantitative relativizations of complexity classes. SIAM J. Comput. 13, 461-487.

Carlyle, J. W. and Paz, A. 1971. Realizations by stochastic finite automata. J. Comput. Syst. Sci. 5, 26-40.

Caro, F. D. 1988. Graded modalities II (canonical models). Studia logica 47, 1-10.

Chandra, A., Kozen, D., And Stockmeyer, L. 1981. Alternation. J. ACM 28, 114-133.

Chellas, B. 1980. Modal Logic. Cambridge University Press, Cambridge.

Cîrstea, C. And Pattinson, D. 2007. Modular construction of complete coalgebraic logics. Theor. Comput. Sci.. In press.

D'Agostino, G. And Visser, A. 2002. Finality regained: A coalgebraic study of Scott-sets and multisets. Arch. Math. Logic 41, 267-298.

De Nivelle, H., Schmidt, R. A., and Hustadt, U. 2000. Resolution-based methods for modal logics. Logic J. IGPL 8, 265-292.

Demri, S. And Lugiez, D. 2006. Presburger modal logic is only PSPACE-complete. In $I J$ CAR 2006, Proceedings of the Third International Joint Conference on Automated Reasoning, U. Furbach and N. Shankar, Eds. Lect. Notes Artificial Intell., vol. 4130. Springer, Berlin, 541-556. Full version available as Research Report LSV-06-15, Laboratoire Spécification et Vérification, Ecole Normale Supérieure de Cachan, 2006.

Emerson, E. A. And Halpern, J. Y. 1985. Decision procedures and expressiveness in the temporal logic of branching time. J. Comput. Syst. Sci. 30, 1-24.

Fagin, R. And Halpern, J. Y. 1994. Reasoning about knowledge and probability. J. ACM 41, $340-367$.

Fine, K. 1972. In so many possible worlds. Notre Dame J. Formal Logic 13, 516-520.

HALPERn, J. AND RÊGO, L. C. 2007. Characterizing the NP-PSPACE gap in the satisfiability problem for modal logic. In IJCAI 2007, Proceedings of the 20th International Joint Conference on Artificial Intelligence, M. M. Veloso, Ed. 2306-2311.

Hansen, H. H. And Kupke, C. 2004. A coalgebraic perspective on monotone modal logic. In Coalgebraic Methods in Computer Science, J. Adámek and S. Milius, Eds. Electron. Notes Theor. Comput. Sci., vol. 106. Elsevier, Amsterdam, 121-143.

Heifetz, A. And Mongin, P. 2001. Probabilistic logic for type spaces. Games and Economic Behavior 35, 31-53.

JACOBS, B. 2000. Towards a duality result in coalgebraic modal logic. In CMCS 2000, Coalgebraic Methods in Computer Science, H. Reichel, Ed. Electron. Notes Theor. Comput. Sci., vol. 33. Elsevier, Amsterdam.

Kupke, C., Kurz, A., And Pattinson, D. 2005. Ultrafilter extensions for coalgebras. In $C A L C O$ 2005, Algebra and Coalgebra in Computer Science: First International Conference, Proceedings, J. L. Fiadeiro, N. Harman, M. Roggenbach, and J. Rutten, Eds. Lect. Notes Comput. Sci., vol. 3629. Springer, Berlin, 263-277.

Kurz, A. 2001. Specifying coalgebras with modal logic. Theor. Comput. Sci. 260, 119-138. 
LADNER, R. 1977. The computational complexity of provability in systems of modal propositional logic. SIAM J. Comput. 6, 467-480.

Larsen, K. And Skou, A. 1991. Bisimulation through probabilistic testing. Inf. Comput. 94, $1-28$.

Mossakowski, T., Schröder, L., Roggenbach, M., And Reichel, H. 2006. Algebraiccoalgebraic specification in CoCASL. J. Logic Algebraic Programming 67, 146-197.

Ohlbach, H. J. And Koehler, J. 1999. Modal logics, description logics and arithmetic reasoning. Artificial Intelligence 109, 1-31.

Pacuit, E. And Salame, S. 2004. Majority logic. In KR 2004, Principles of Knowledge Representation and Reasoning: Proceedings of the Ninth International Conference, D. Dubois, C. A. Welty, and M.-A. Williams, Eds. AAAI Press, 598-605.

Pattinson, D. 2001. Semantical principles in the modal logic of coalgebras. In STACS 2001, 18th Annual Symposium on Theoretical Aspects of Computer Science, Proceedings, A. Ferreira and H. Reichel, Eds. Lect. Notes Comput. Sci., vol. 2010. Springer, Berlin, 514-526.

PAttinson, D. 2003. Coalgebraic modal logic: Soundness, completeness and decidability of local consequence. Theor. Comput. Sci. 309, 177-193.

Pattinson, D. 2004. Expressive logics for coalgebras via terminal sequence induction. Notre Dame J. Formal Logic 45, 19-33.

PAUly, M. 2002. A modal logic for coalitional power in games. J. Logic and Comput. 12, 149-166.

PAUly, M. 2005. On the role of language in social choice theory. Unpublished manuscript.

Rabin, M. 1963. Probabilistic automata. Inform. Control 6, 230-245.

Rössiger, M. 2000. Coalgebras and modal logic. In CMCS 2000, Coalgebraic Methods in Computer Science, H. Reichel, Ed. Electron. Notes Theor. Comput. Sci., vol. 33. Elsevier, Amsterdam.

Rothe, J., Tews, H., And Jacobs, B. 2001. The Coalgebraic Class Specification Language CCSL. J. Universal Comput. Sci. 7, 175-193.

Rutten, J. 2000. Universal coalgebra: A theory of systems. Theor. Comput. Sci. 249, 3-80.

SchriJver, A. 1986. Theory of linear and integer programming. John Wiley \& Sons, Chichester.

SCHRÖDER, L. 2005. Expressivity of coalgebraic modal logic: the limits and beyond. In FOSSACS 2005, Foundations of Software Science and Computation Structures, 8th International Conference, Proceedings, V. Sassone, Ed. Lect. Notes Comput. Sci., vol. 3441. Springer, Berlin, 440-454. Extended version to appear in Theor. Comput. Sci.

SChröDer, L. 2007. A finite model construction for coalgebraic modal logic. J. Logic Algebraic Programming. In press. Earlier version in Foundations of Software Science And Computation Structures, vol. 3921 of Lect. Notes Comput. Sci., pp. 157-171, Springer, Berlin, 2006.

Schröder, L. And Pattinson, D. 2006. PSPACE reasoning for rank-1 modal logics. In LICS 2006, Proceedings of the 21st Annual IEEE Symposium on Logic in Computer Science, R. Alur, Ed. IEEE Computer Society Press, 231-240. Presentation slides available under www.informatik.uni-bremen.de/ lschrode/slides/rank1pspace.pdf.

Schröder, L. And PAttinson, D. 2007a. Modular algorithms for heterogeneous modal logics. In ICALP 2007, Automata, Languages and Programming, 34th International Colloquium, Proceedings, L. Age, A. Tarlecki, and C. Cachin, Eds. Lect. Notes Comput. Sci. Springer, Berlin. To appear.

SChröDer, L. And PAtTinson, D. 2007b. Rank-1 modal logics are coalgebraic. In STACS 2007, 24 th Annual Symposium on Theoretical Aspects of Computer Science, Proceedings, W. Thomas and P. Weil, Eds. Lect. Notes Comput. Sci., vol. 4393. Springer, Berlin, 574-585.

ToBies, S. 2001. PSPACE reasoning for graded modal logics. J. Logic and Comput. 11, 85-106.

Troelstra, A. S. and Schwichtenberg, H. 1996. Basic Proof Theory. Cambridge Tracts in Theoretical Computer Science, vol. 43. Cambridge University Press, Cambridge.

Turi, D. And Plotkin, G. 1997. Towards a mathematical operational semantics. In LICS 1997, Proceedings of the 12th Annual IEEE Symposium on Logic in Computer Science. IEEE Computer Society Press, 280-291. 
VAN Der Hoek, W. And Meyer, J.-J. 1992. Graded modalities in epistemic logic. In LFCS 1992, Logical Foundations of Computer Science, Second International Symposium, Proceedings, A. Nerode and M. A. Taitslin, Eds. Lect. Notes Comput. Sci., vol. 620. Springer, Berlin, 503514

VARDI, M. 1989. On the complexity of epistemic reasoning. In LICS 1989, Proceedings of the Fourth Annual IEEE Symposium on Logic in Computer Science. IEEE Computer Society Press, 243-251.

VARDI, M. Y. 1996. Why is modal logic so robustly decidable? In Descriptive Complexity and Finite Models, Proceedings of a DIMACS Workshop, N. Immerman and P. G. Kolaitis, Eds. DIMACS Ser. in Discrete Math. and Theor. Comput. Sci., vol. 31. American Mathematical Society, 149-184.

Venema, Y. 2006. Automata and fixed point logics: a coalgebraic perspective. Inf. Comput. 204, 637-678. 